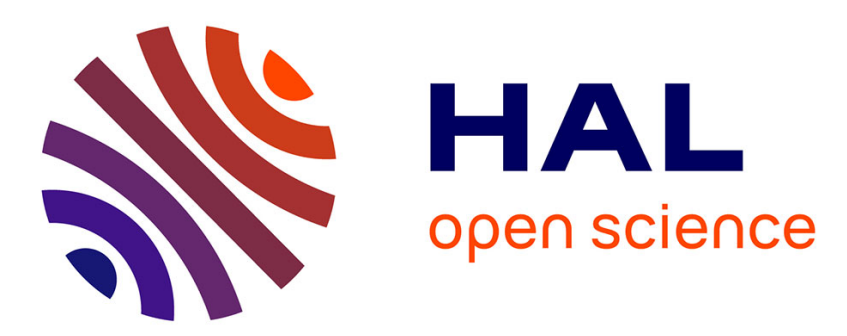

\title{
Uvéites sous biothérapies et/ou DMARDS : analyse de la base de données déclarative de la pharmacovigilance nationale française
}

Laura Cabane

\section{- To cite this version:}

Laura Cabane. Uvéites sous biothérapies et/ou DMARDS : analyse de la base de données déclarative de la pharmacovigilance nationale française. Médecine humaine et pathologie. 2016. dumas-01407755

\section{HAL Id: dumas-01407755 https://dumas.ccsd.cnrs.fr/dumas-01407755}

Submitted on 2 Dec 2016

HAL is a multi-disciplinary open access archive for the deposit and dissemination of scientific research documents, whether they are published or not. The documents may come from teaching and research institutions in France or abroad, or from public or private research centers.
L'archive ouverte pluridisciplinaire HAL, est destinée au dépôt et à la diffusion de documents scientifiques de niveau recherche, publiés ou non, émanant des établissements d'enseignement et de recherche français ou étrangers, des laboratoires publics ou privés. 
Université de Nice Sophia - Antipolis

Faculté de Médecine de Nice

Année 2015- 2016

\section{Uvéites sous biothérapies et/ou DMARDS : Analyse de la base de données déclarative de la pharmacovigilance nationale française.}

THESE DE MEDECINE

Pour l'obtention du grade de Docteur en Médecine (Diplôme d'état)

Présentée et soutenue publiquement le jeudi 22 septembre 2016 par

\section{Laura CABANE}

Née le 26 Février 1988 à Nice

Interne des Hôpitaux de Nice

Spécialité Rhumatologie

Composition du jury:

Madame le Professeur Liana EULLER-ZIEGLER

Madame le Professeur Stéphanie BAILLIF

Madame le Professeur Véronique BREUIL

Monsieur le Professeur Christian ROUX

Madame le Docteur Nathalie TIEULIE
Présidente du jury

Assesseur

Assesseur

Assesseur

Directrice de thèse 
Liste des professeurs au 1er septembre 2016 à la Faculté de Médecine de Nice

Doyen

Vice-Doyen

Assesseurs

Conservateur de la bibliothèque

Directrice administrative des services

Doyens Honoraires

\section{BAQUÉ Patrick}

M. BOILEAU Pascal

M. ESNAULT Vincent

M. CARLES Michel

Mme BREUIL Véronique

M. MARTY Pierre

Mme DE LEMOS Annelyse

Mme CALLEA Isabelle

M. AYRAUD Noël

M. RAMPAL Patrick

M. BENCHIMOL Daniel

Professeurs Honoraires

M ALBERTINI Marc

M. BALAS Daniel

M. BATT Michel

M. BLAIVE Bruno

M. BOQUET Patrice

M. BOURGEON André

M. BOUTTÉ Patrick

M. BRUNETON Jean-Noël

Mme BUSSIERE Françoise

M. CAMOUS Jean-Pierre

M. CANIVET Bertrand

M. CASSUTO Jill-patrice

M. CHATEL Marcel

M. COUSSEMENT Alain

Mme CRENESSE Dominique

M. DARCOURT GUY

M. DELLAMONICA Pierre

M. DELMONT Jean

M. DEMARD François

M. DOLISI Claude

M . FRANCO Alain

M. FREYCHET Pierre

M. GÉRARD Jean-Pierre

M. GILLET Jean-Yves

M. GRELLIER Patrick

M. GRIMAUD Dominique
M. HARTER Michel

M. INGLESAKIS Jean-André

M. JOURDAN Jacques

M. LALANNE Claude-Michel

M. LAMBERT Jean-Claude

M. LAZDUNSKI Michel

M. LEFEBVRE Jean-Claude

M. LE BAS Pierre

M. LE FICHOUX Yves

Mme LEBRETON Elisabeth

M. LOUBIERE Robert

M. MARIANI Roger

M. MASSEYEFF René

M. MATTEI Mathieu

M. MOUIEL Jean

Mme MYQUEL Martine

M. OLLIER Amédée

M. ORTONNE Jean-Paul

M. SAUTRON Jean Baptiste

M. SCHNEIDER Maurice

M. TOUBOL Jacques

M. TRAN Dinh Khiem

M VAN OBBERGHEN Emmanuel

M. ZIEGLER Gérard 
Liste des professeurs au 1er septembre 2016 à la Faculté de Médecine de Nice

M. ARNOLD Jacques
M. BASTERIS Bernard
MIle CHICHMANIAN Rose-Marie
Mme DONZEAU Michèle
M. EMILIOZZI Roméo
M. FRANKEN Philippe
M. GASTAUD Marcel
M.GIRARD-PIPAU Fernand
M. GIUDICELLI Jean
M. MAGNÉ Jacques
Mme MEMRAN Nadine
M. MENGUAL Raymond
M. PHILIP Patrick
M. POIRÉE Jean-Claude
Mme ROURE Marie-Claire




\section{PROFESSEURS CLASSE EXCEPTIONNELLE}

M. AMIEL Jean

M. BENCHIMOL Daniel

M. BOILEAU Pascal

M. DARCOURT Jacques

M. DESNUELLE Claude

Mme EULLER-ZIEGLER Liana

M. FENICHEL Patrick

M. FUZIBET Jean-Gabriel

M. GASTAUD Pierre

M. GILSON Éric

M. HASSEN KHODJA Reda

M. HÉBUTERNE Xavier

M. HOFMAN Paul

M. LACOUR Jean-Philippe

M. LEFTHERIOTIS Geogres

M. MARTY Pierre

M. MICHIELS Jean-François

M. MOUROUX Jérôme

Mme PAQUIS Véronique

M. PAQUIS Philippe

M. PRINGUEY Dominique

M. QUATREHOMME Gérald

M. RAUCOULES-AIMÉ Marc

M. ROBERT Philippe

M. SANTINI Joseph

M. THYSS Antoine

M. TRAN Albert
Urologie (52.04)

Chirurgie Générale (53.02)

Chirurgie Orthopédique et Traumatologique (50.02)

Biophysique et Médecine Nucléaire (43.01)

Biologie Cellulaire (44.03)

Rhumatologie (50.01)

Biologie du Développement et de la Reproduction (54.05)

Médecine Interne (53.01)

Ophtalmologie (55.02)

Biologie Cellulaire (44.03)

Chirurgie Vasculaire (51.04)

Nutrition (44.04)

Anatomie et Cytologie Pathologiques (42.03)

Dermato-Vénéréologie (50.03)

Physiologie- médecine vasculaire

Parasitologie et Mycologie (45.02)

Anatomie et Cytologie Pathologiques (42.03)

Chirurgie Thoracique et Cardiovasculaire (51.03)

Génétique (47.04)

Neurochirurgie (49.02)

Psychiatrie d'Adultes (49.03)

Médecine Légale et Droit de la Santé (46.03)

Anesthésie et Réanimation Chirurgicale (48.01)

Psychiatrie d'Adultes (49.03)

O.R.L. (55.01)

Cancérologie, Radiothérapie (47.02)

Hépato Gastro-entérologie (52.01) 


\section{Liste des professeurs au 1er septembre 2016 à la Faculté de Médecine de Nice}

\section{PROFESSEURS PREMIERE CLASSE}

Mme ASKENAZY-GITTARD Florence

M. BAQUÉ Patrick

M. BARRANGER Emmanuel

M. BÉRARD Étienne

M. BERNARDIN Gilles

Mme BLANC-PEDEUTOUR Florence

M. BONGAIN André

M. CASTILLO Laurent

M. DE PERETTI Fernand

M. DRICI Milou-Daniel

M. ESNAULT Vincent

M. FERRARI Émile

M. FERRERO Jean-Marc

M. GIBELIN Pierre

M. GUGENHEIM Jean

M. HANNOUN-LEVI Jean-Michel

Mme ICHAI Carole

M. LONJON Michel

M. MARQUETTE Charles-Hugo

M. MOUNIER Nicolas

M. PADOVANI Bernard

M. PRADIER Christian

Mme RAYNAUD Dominique

M. ROSENTHAL Éric

M. SCHNEIDER Stéphane

M. STACCINI Pascal

M. THOMAS Pierre
Pédopsychiatrie (49.04)

Anatomie - Chirurgie Générale (42.01)

Gynécologie Obstétrique (54.03)

Pédiatrie (54.01)

Réanimation Médicale (48.02)

Cancérologie - Génétique (47.02)

Gynécologie-Obstétrique (54.03)

O.R.L. (55.01)

Anatomie-Chirurgie Orthopédique (42.01)

Pharmacologie Clinique (48.03)

Néphrologie (52-03)

Cardiologie (51.02)

Cancérologie ; Radiothérapie (47.02)

Cardiologie (51.02)

Chirurgie Digestive (52.02)

Cancérologie ; Radiothérapie (47.02)

Anesthésiologie et Réanimation Chirurgicale (48.01)

Neurochirurgie (49.02)

Pneumologie (51.01)

Cancérologie, Radiothérapie (47.02)

Radiologie et Imagerie Médicale (43.02)

Épidémiologie, Économie de la Santé et Prévention (46.01)

Hématologie (47.01)

Médecine Interne (53.01)

Nutrition (44.04)

Biostatistiques et Informatique Médicale (46.04)

Neurologie (49.01) 
Liste des professeurs au 1er septembre 2016 à la Faculté de Médecine de Nice

\section{PROFESSEURS DEUXIEME CLASSE}

M. BAHADORAN Philippe

Mme BAILLIF Stéphanie

M. BENIZRI Emmanuel

M. BENOIT Michel

M. BREAUD Jean

Mlle BREUIL Véronique

M. CARLES Michel

M. CHEVALIER Nicolas

M. CHEVALLIER Patrick

Mme CHINETTI Giulia

M. DELLAMONICA Jean

M. DELOTTE Jérôme

M. FONTAINE Denys

M. FOURNIER Jean-Paul

Mlle GIORDANENGO Valérie

M. GUÉRIN Olivier

M. IANNELLI Antonio

$M \quad J E A N$ BAPTISTE Elixène

M. LEVRAUT Jacques

M. PASSERON Thierry

M. PICHE Thierry

M. ROGER Pierre-Marie

M. ROHRLICH Pierre

M. ROUX Christian

M. RUIMY Raymond

Mme SACCONI Sabrina

M. SADOUL Jean-Louis

M. TROJANI Christophe

M. VENISSAC Nicolas
Cytologie et Histologie (42.02)

Ophtalmologie (55.02)

Chirurgie Générale (53.02)

Psychiatrie (49.03)

Chirurgie Infantile (54-02)

Rhumatologie (50.01)

Anesthésiologie Réanimation (48.01)

Endocrinologie, Diabète et Maladies Métaboliques (54.04)

Radiologie et Imagerie Médicale (43.02)

Biochimie-Biologie Moléculaire (44.01)

réanimation médicale (48.02)

Gynécologie-obstétrique (54.03)

Neurochirurgie (49.02)

Thérapeutique (48-04)

Bactériologie-Virologie (45.01)

Gériatrie (48.04)

Chirurgie Digestive (52.02)

Chirurgie vasculaire (51.04)

Anesthésiologie et Réanimation Chirurgicale (48.01)

Dermato-Vénéréologie (50-03)

Gastro-entérologie (52.01)

Maladies Infectieuses; Maladies Tropicales (45.03)

Pédiatrie (54.01)

rhumatologie (50.01)

Bactériologie-virologie (45.01)

Neurologie (49.01)

Endocrinologie, Diabète et Maladies Métaboliques (54.04)

Chirurgie Orthopédique et Traumatologique (50.02)

Chirurgie Thoracique et Cardiovasculaire (51.03) 
Liste des professeurs au 1er septembre 2016 à la Faculté de Médecine de Nice

\section{PROFESSEUR DES UNIVERSITÉS}

M. HOFLIGER Philippe

Médecine Générale

\section{PROFESSEURS AGRÉGÉS}

Mme LANDI Rebecca Anglais

Mme ROSE Patricia Anglais

\section{MAITRES DE CONFÉRENCES DES UNIVERSITÉS - PRATICIENS HOSPITALIERS}

Mme ALUNNI Véronique

M. AMBROSETTI Damien

Mme BANNWARTH Sylvie

M. BENOLIEL José

Mme BERNARD-POMIER Ghislaine

Mme BUREL-VANDENBOS Fanny

M. DARMON David

M. DOGLIO Alain

$M \quad$ DOYEN Jérôme

M FAVRE Guillaume

M. FOSSE Thierry

M. GARRAFFO Rodolphe

Mme GIOVANNINI-CHAMI Lisa

Mme HINAULT Charlotte

M. HUMBERT Olivier

Mme LAMY Brigitte

Mme LEGROS Laurence

Mme LONG-MIRA Elodie

Mme MAGNIÉ Marie-Noëlle

Mme MOCERI Pamela

Mme MUSSO-LASSALLE Sandra

M. NAÏMI Mourad

Mme POMARES Christelle

Mme SEITZ-POLSKI barbara

M. TESTA Jean

M. TOULON Pierre
Médecine Légale et Droit de la Santé (46.03)

Cytologie et Histologie (42.02)

Génétique (47.04)

Biophysique et Médecine Nucléaire (43.01)

Immunologie (47.03)

Anatomie et Cytologie pathologiques (42.03)

Médecine Générale

Bactériologie-Virologie (45.01)

Radiothérapie (47.02)

Néphrologie (52.03)

Bactériologie-Virologie-Hygiène (45.01)

Pharmacologie Fondamentale (48.03)

Pédiatrie (54.01)

Biochimie et biologie moléculaire (44.01)

Biophysique et Médecine Nucléaire (43.01)

Bactérilogie-virologie ( 45.01)

Hématologie et Transfusion (47.01)

Cytologie et Histologie (42.02)

Physiologie (44.02)

Cardiologie (51.02)

Anatomie et Cytologie pathologiques (42.03)

Biochimie et Biologie moléculaire (44.01)

Parasitologie et mycologie (45.02)

Immunologie (47.03)

Épidémiologie Économie de la Santé et Prévention (46.01)

Hématologie et Transfusion (47.01) 
Liste des professeurs au 1er septembre 2016 à la Faculté de Médecine de Nice

\section{PRATICIEN HOSPITALIER UNIVERSITAIRE}

M. DURAND Matthieu

Urologie (52.04)

\section{PROFESSEURS ASSOCIÉS}

M. GARDON Gilles

M. GONZALEZ Jean-François

M. PAPA Michel

M. WELLS Michael

\section{MAITRES DE CONFÉRENCES ASSOCIÉS}

M BALDIN Jean-Luc

Mme CASTA Céline

Mme MONNIER Brigitte
Médecine Générale

Chirurgie Orthopédique et traumatologie ( 50.02)

Médecine Générale

Anatomie-Cytologie ( 42.03)

Médecine Générale

Médecine Générale

Médecine Générale

\section{PROFESSEURS CONVENTIONNÉS DE L'UNIVERSITÉ}

M. BERTRAND François

M. BROCKER Patrice

M. CHEVALLIER Daniel

Mme FOURNIER-MEHOUAS Manuella

M. JAMBOU Patrick

M. ODIN Guillaume

M. PEYRADE Frédéric

M. PICCARD Bertrand

M. QUARANTA Jean-François
Médecine Interne

Médecine Interne Option Gériatrie

Urologie

Médecine Physique et Réadaptation

Coordination prélèvements d'organes

Chirurgie maxilo-faciale

Onco-Hématologie

Psychiatrie

Santé Publique 


\section{Remerciements}

A mes parents,

Merci d'avoir fait de moi ce que je suis devenue. J'étais fière de lire mon prénom sur vos deux thèses et je le suis encore plus de pouvoir vous présenter la mienne.

A mes grands-parents,

J'espère que vous êtes fiers de moi et que toi, Papito, tu me regardes de là-haut...

A ma grande famille extraordinaire,

Ma tribu de frères et sœurs, ma horde de cousins, les tatas et les tontons. Je suis très fière de faire partie de cette heureuse et grande famille. Je vous aime.

A mon mari, mon amoureux, mon tout.

Merci d'être dans ma vie depuis presque 10 ans, d'être là dans les moments de joie comme dans les moments de tristesse pour me soutenir et me porter toujours plus loin. Je t'aime.

A mes amis depuis maintenant une bonne décennie, Sonia mon petit chat noir, Eve " ma Tara ", Clemou, Plattoche, Kiwi, Roneiiiiil (vive le trouple !!), Ben, les Floje, Anaïs, PM, David, Kevin, Emma, Meylie... et toutes vos " pièces rapportées » devenues des supers copains. Kiss love cœur carré carré point !

A mes amies d'enfance : Bibich et Lau. Je vous aime.

A la team du bureau de l'Internat, parce que la soirée ne fait que commencer !!!!! : Mélo, Gégé, Marc, Jeff, Julien, Ben, Rémi, Ghis et Pierre. 
A Madame le Professeur Euller-Ziegler, Merci de m'avoir fait l'honneur de présider mon jury et de m'avoir permis de réaliser ce travail. Vos conseils tout au long de mon internat ont été précieux. Veuillez recevoir dans cette thèse le témoignage de ma profonde reconnaissance.

A Madame le Professeur Breuil, Merci pour ton implication dans ma thèse, ton soutien et ton encadrement tout au long de mon cursus universitaire. Je suis fière que tu $\mathrm{m}^{\prime}$ accordes ta confiance afin de continuer à travailler à tes côtés en tant que docteur en rhumatologie.

A Madame le Professeur Baillif, Je vous remercie d'avoir accepté de juger ce travail. Je suis honorée de votre présence dans mon jury de thèse. Veuillez recevoir le témoignage de mon profond respect.

A Monsieur le Professeur Roux, Je suis contente que tu aies accepté de siéger dans ce jury. Merci pour le soutien psychologique pré-thèse et pour le " comment avancer sa thèse au mois d'aout ". Merci également pour ton encadrement tout au long de ces années et d'avoir partagé un peu de tes consultations avec moi.

A Docteur Bernadette Baldin, sans qui cette thèse n'aurait pas pu voir le jour. Merci pour votre gentillesse et votre grande disponibilité.

Au Docteur Nathalie Tieulié, Merci pour la confiance que tu m'as accordée en acceptant d'encadrer ma thèse. Tu es pour moi un modèle, un médecin incroyable et je tiens à te remercier pour tout ce que tu as fait pour moi tant au niveau professionnel que personnel. Ton investissement auprès des patients est tel qu'il nous pousse à toujours faire mieux. Je suis fière de pouvoir travailler à tes cotés. 
A ma co-interne de rhumatologie, Lindsay, ma copine, pour toutes ces années d'internat qu'on a partagé mais aussi pour tous ces moments à côté. J'espère qu'on continuera encore longtemps à avoir « envie de se rouler par terre »!! pffffiouuuuuuuuu.

A mes autres co-internes de rhumato avec qui j'ai passé de très bons semestres : Adeline et son fameux cours de ****, Elsa Lancelot, scintillante Lauren, Béné Minimoi, Alex notre seul mec ;), et nos bb aurore et Stéphanie $\odot$. J'attends toujours le cours de DES-apéro-mojito-Plazza !!

A mes co-internes non rhumato:

Laurie " Ginette ", Sam (maitre pokemon! :p) et Margaux chou pour ce deuxième semestre de folie mais aussi pour toutes les autres années qui ont suivi et qui suivront ! « Pompelup »!!!

Faustine et Dianou pour ce troisième semestre " girls only »

Julia mon binôme, Camille « pony pony run run » et Denis la malice pour mon $5^{\text {ème }}$ semestre.

Violaine mon petit bourdon et JuJu, membres du covoiturage du feu de dieu ; Micha (j'ai adoré trouver des \#maladiesquepersonneconnait avec toi !) pour ce dernier semestre.

Au service de rhumatologie de l'Archet 1 puis de Pasteur 2 qui m'a vu évoluer durant mon internat :

- L'HT et son équipe, notamment Mickey, Yacine, Laura, Anne-So, Camille, Clémence, angé, Céline notre cadre de choc, mais aussi Delphine, Marianne, Cédric, Pierre, Franck et tous les autres !

A Emilie et Amélie : merci pour les semestres passés ensemble. J'ai beaucoup appris à vos côtés, j'ai aussi beaucoup rigolé. Vous êtes un peu des modèles pour nous et je suis très contente de pouvoir vous compter parmi mes chefs. 
A Christine : tu as été ma première chef de rhumatologie. Merci pour ta rigueur professionnelle et ton encadrement. J'ai beaucoup aimé travailler avec toi.

A Philippe : un grand merci pour tes conseils, ton enseignement allant de la rhumatologie aux langues étrangères, mais aussi pour ta bonne humeur à toute épreuve et les super pan bagnats party. Je vous souhaite encore beaucoup de voyages et de parcours de golf avec Annie!

A Aurore : tu n'as pas été ma chef longtemps mais quelques jours à tes côtés ont suffi à me montrer quelle clinicienne tu es. Merci de le faire partager.

- L'HDS et ses trois piliers : Isa, Virginie et Brigitte. Egalement, Danielle, Edith, Nathalie et Patricia.

A Christian Grisot : on dit qu'il y a des personnalités qui marquent une vie. Et bien tu en fais partie. Merci d'avoir cru en moi et de m'avoir poussé toujours plus loin. « Lolo, la rhumato $c^{\prime}$ est pas que les anticorps anti poils de $* * *$ ! Va chercher le podoscope! ». Je n'oublierai pas. Merci.

- L'HDJ et ses viennoiseries $;:$ : Cindy, Anne, chrystel, Cathy et chantal.

- Aux docteurs Hasna Chami et Yacine Allam.

- A nos secrétaires chéries qui ont adoré taper nos compte-rendus hihi : Audrey, Geneu, Marine, Véro, Mélissa. Merci pour votre patience.

- A Nathalie Piche, merci pour ton aide précieuse pendant ma pêche aux articles !!

- A nos kinés, notamment irremplaçable Gérald!

- A nos visiteurs médicaux préférés : Christelle, Didier et Nico (promis on ira faire un karaoké !!) 
A l'ensemble du service d'hématologie du Pr Rohrlich : premier stage en tant qu'interne...

Merci particulièrement Pr Mounier pour votre enseignement et votre rigueur, $\mathrm{Dr}$ Karsenti pour toutes les gardes passées ensemble à Saint Roch et Dr Vinti pour votre patience et votre bonne humeur à toute épreuve. Ça a été un plaisir de travailler avec vous et de débuter mon internat encadrée par une super équipe.

Merci aux IDE : Mélanie petit chat noir, maman Laeti, Dédé, François, Eva, Philippe, Pauline... mais aussi à tous les AS, ASH, secrétaires, psychologues et à notre Sophie la diet'.

Merci aussi à mon équipe de choc : ma Valou et Ines pour tous ces moments passés ensemble : la première PL (ah non pardon, chimio IT !), le premier myélo, la première annonce... et j'en passe.

Au Pr Fuzibet et à l'ensemble du service de médecine interne de l'Archet 1.

A Viviane Queyrel, pour sa gentillesse, son encadrement.

J'ai appris énormément à tes côtés, et pas seulement sur la médecine. Tu es une femme incroyable, merci Viviane.

A Eléa, tu es un médecin brillant et une petite boule d'énergie.

J'ai été fière d'avoir été ton interne mais également ta patiente ;). Merci.

A mes co-internes, Gwendo, Nihal (le modèle indien ;)), Maxime, Marion et Xavier

Merci particulièrement à l'équipe du A avec qui j'ai passé 6 mois incroyables, notamment Jess, aurore et Céline mes petites reines de la piqure:-), Nadège, Marylène, Sandrine, Mumu, Véro et toutes les autres...

Un remerciement spécial pour Domi qui a écouté toutes nos bêtises dans le dictaphone pendant tout un semestre ! 
A l'équipe d'onco-hématologie pédiatrique de l'Archet 2 du Pr Rohrlich,

L'équipe médicale : Joy, Françoise, Maryline, Fabrice, Anne et Marion, mais également paramédicale qui est au top ! J'ai beaucoup aimé mon passage dans le service, votre courage et votre dévouement envers les enfants.

A Christine Soler, merci de m'avoir acceptée à tes côtés et d'avoir partagé avec moi ton expérience sur cette spécialité qui me tient tant à cœur. J'ai adoré travailler avec toi et j'espère pouvoir continuer à le faire.

A mon co-interne, mon petit Juju. Je garderai un très bon souvenir de nos batailles d’eau (voir d'autres choses ?! ^^) au milieu du service et de nos déménagements de dossiers pour le staff !

A tous les enfants dont j'ai croisé la route durant ces 6 mois : Carla, Valentin, Charlotte notamment. Vous m'avez appris à relativiser, à être plus forte. Une belle leçon de vie, je n'oublierai pas.

Au service de dermatologie de l'Archet 2 et à sa belle équipe Aux Pr Lacour et Passeron, pour leur enseignement si passionnant.

A Marine, princesse de la pustule ! :) Merci pour ces 6 mois à tes côtés en HDJ.

A Henri, (prince du mélanome ?^^$^{\wedge}$ ) pour ta rigueur et ta gentillesse.

A Fériel, pour ta bonne humeur et tout ce que tu fais partager aux autres.

A Olivia, j'ai adoré bossé avec toi, ne change pas!

A toute l'équipe soignante : Laura (mon double), margaux, Elsa, Célia, Sophie, Vanessa, Karen, les 2 Julie, Stéph, Magalie, Isa, Sandra et toutes les autres.

A mes super co-internes du pays des bisounours : Alex (hâte de te retrouver chef en Novembre !), Sophie (j'espère que tu as trouvé quelqu'un pour faire tes PASI du dimanche :p), Alicia (ma copine de sport), Pierre-Mich' alias le fuego, Charline, Ahmad, Azzam, Davide, Hélène (dans son antre !). A nos petites réunions sponsorisées et nos apéros! 
A Johan, Cécile et Karine qui m'ont fait un peu vivre un stage d'infectiologie par procuration;)

A mes autres copains internes: Anne, Audrey L, Charlotte, Céline, Adrien, Coralie, clémence, Cécile, Agnès, Gautier, Audrey H, Victoria, Elodie... et j'en passe :)

A tous mes externes,

Particulièrement à l'équipe de choc : Mathilde, Laura, Marine, Marie-Charlotte, Samantha, Alister, colline, Mathilde...

Merci à tous ceux qui m'ont accueilli pour un café lors de mes gardes à l'Archet :

I'infectiologie et son fameux Cappu' (Amandine, Emeline, Maryline, Sarah, Christophe, Olivier, Valérie...), I’hématologie et la médecine interne.

Enfin, merci à tous ceux qui ont fait un bout de chemin avec moi et que j'ai probablement oublié de citer.

"Soyons reconnaissants aux personnes qui nous donnent du bonheur ; elles sont les charmants jardiniers par qui nos âmes sont fleuries."

Marcel Proust 


\section{Table des matières}

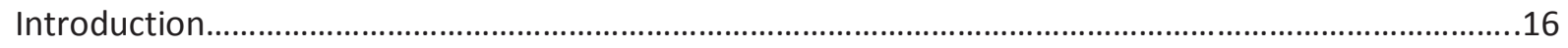

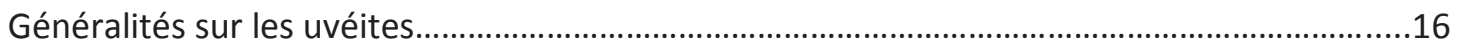

Uvéites et rhumatismes inflammatoires...........................................................................18

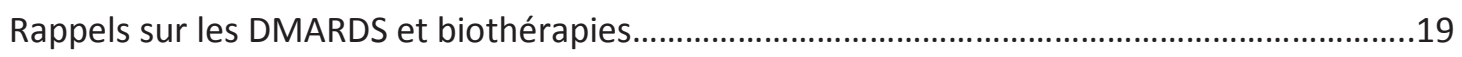

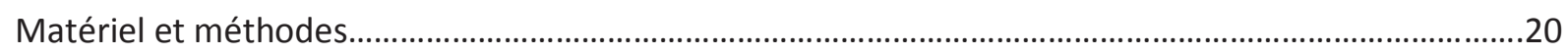

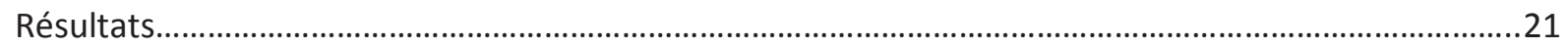

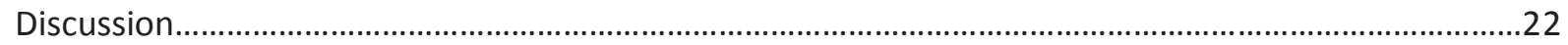

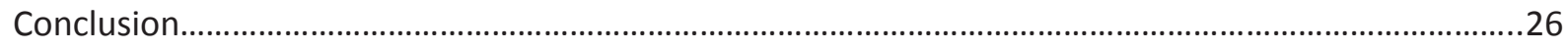

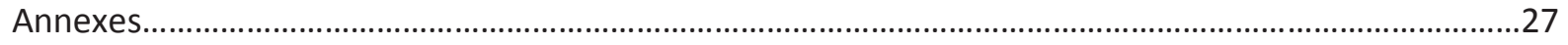

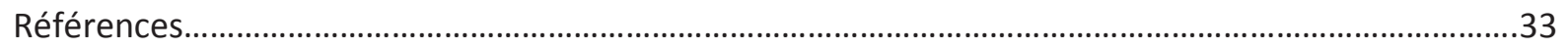

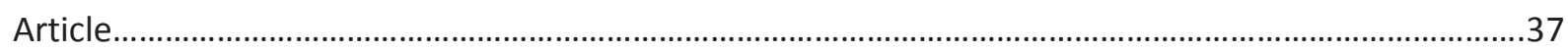

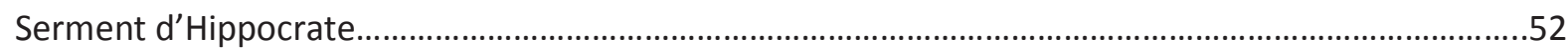




\section{Introduction}

Face à la survenue d'une uvéite chez les patients traités par biothérapie et/ou DMARDs, le rhumatologue doit en préciser rapidement l'origine pour optimiser la prise en charge. L'uvéite est une inflammation intra-oculaire qui peut représenter une menace pour le pronostic visuel des patients. Les uvéites représentent $10 \%$ des causes de cécité dans les pays industrialisés. ${ }^{1,2}$ La nécessité d'un diagnostic précis chez un patient traité pour un rhumatisme inflammatoire chronique vient du fait que les uvéites peuvent être d'origine inflammatoire, en rapport avec la maladie sous-jacente (la spondylarthrite ankylosante est associée dans 5 à $15 \%$ des cas à des uvéites spécifiques. ${ }^{3,4}$ ), mais également d'origine infectieuse, favorisée par l'immunodépression liée à la biothérapie et/ou aux DMARDs. A ce jour, nous ne disposons pas de données sur les étiologies des uvéites chez des patients traités par biothérapie et/ou DMARDs pour une maladie inflammatoire chronique.

Le but de notre travail a été :

- de décrire le type d'uvéites apparues chez des patients présentant un rhumatisme inflammatoire ou une maladie chronique de l'intestin (MICl) ou dermatologique (psoriasis), traités par biothérapie et/ou DMARDS et ayant fait l'objet d'une déclaration à la pharmacovigilance

- d'évaluer quelle proportion d'uvéites correspond à une poussée spécifique de la maladie, quelle proportion correspond à une complication infectieuse et si un effet secondaire direct du médicament peut être retenue comme étiologie.

\section{Généralités sur les uvéites}

La prise en charge des uvéites repose avant tout sur leur présentation anatomique. ${ }^{5-8}$

On distingue :

- les uvéites antérieures (UA) atteignant l'iris (iritis), le corps ciliaire (cyclite) ou les deux (iridocyclite). 
- les uvéites intermédiaires (UI), correspondant à une inflammation du vitré (hyalite).

- les uvéites postérieures (UP) touchant la choroïde voire même la rétine (choriorétinite).

- la panuvéite (panUV) qui est une inflammation touchant toutes les structures de l'uvée.

Dans la population générale, les uvéites font partie d'un groupe de maladies hétérogènes et complexes, représentant un véritable défi diagnostique et thérapeutique pour les praticiens. Les données épidémiologiques ont longtemps été rares et incomplètes en raison du manque de définition commune au terme uvéite et de ses différentes entités cliniques. L'International Uveitis Study Group a toutefois permis une standardisation des descriptions cliniques ${ }^{9}$ (Table I). Les uvéites surviennent à tout âge avec un sex ratio de $1^{10-13}$. Leur incidence est estimée à 17 à 32/100 000 habitants par an et leur prévalence serait de 38 à 204/100 000 habitants, ce qui correspond à une incidence de 9350-13 200 cas par an en France toutes causes confondues ${ }^{10-13}$. Dans les pays occidentaux, les UA sont les formes anatomiques les plus fréquentes (45 à $61 \%$ ), suivies par les UP (13 à 26\%) puis par les UI (8 à 22\%). Les panuvéites représentent quant à elles 7 à $20 \%$ des cas dans les séries ${ }^{5}$.

L'examen clinique ophtalmologique et la description de l'uvéite doivent répondre à ces questions sur ses caractéristiques biomicroscopiques : l'uvéite est-elle granulomateuse ? Unilatérale stricte ou bilatérale ? L'uvéite est-elle hypertensive ? Synéchiante ? Ceci permet d'orienter le diagnostic étiologique (Schéma 1).

D'un point de vue étiologique, parmi les uvéites antérieures, les causes infectieuses dominent avec les uvéites du groupe herpès à HSV et VZV (31,1\%) qui sont les plus fréquemment observées. Ce sont des uvéites unilatérales le plus souvent, granulomateuses, hypertensives et non synéchiantes ${ }^{5,14}$. La cyclite de Fuchs est retrouvée dans 9,5\% mais serait elle-même liée à I'herpès. Les uvéites liées à HLAB27 avec ou sans rhumatisme associé représentent $17,4 \%$ des atteintes. Viennent ensuite les uvéites de la sarcoïdose $(6,8 \%)$ et la tuberculose $(4,9 \%)$. Les formes idiopathiques représentent quant à elles $13,6 \%$ des uvéites antérieures ${ }^{5,14}$ (Figure 1). 
Les uvéites intermédiaires sont majoritairement idiopathiques $(75,5 \%)$ dans le monde. Parmi les causes infectieuses, il convient de citer la maladie de Lyme (2,9\%), la tuberculose $(1,4 \%)$, la rickettsiose $(1,4 \%)$ et la syphilis ${ }^{5,14}$. En Europe, les deux principales causes non infectieuses sont la sclérose en plaque $(10,8 \%)$ et la sarcoïdose $(2,9 \%)$ (Figure 2$)$.

La première cause d'uvéite postérieure est infectieuse : la choriorétinite toxoplasmique (39\%) qui se traduit en général par un foyer systématisé laissant une cicatrice sur la rétine ${ }^{15}$. On note également la tuberculose $(0,5 \%)$, la syphilis $(3 \%)$ et la rétinite à $\mathrm{CMV}$, derrière les formes idiopathiques $(16,5 \%)$ et les causes plus rares telles que la choriorétinopathie de Birdshot, la sarcoïdose $(1,5 \%)$ et la maladie de Behçet $(5 \%)^{5,14,15}$ (Figure 3).

Concernant les panuvéites, ce sont les formes anatomiques les moins fréquentes. Les causes infectieuses sont représentées principalement par la tuberculose $(6,8 \%)$, la toxoplasmose oculaire $(9,9 \%)$ et les rétinites virales nécrosantes. Les causes idiopathiques sont les plus souvent retrouvées $(37,6 \%)$ avant les causes liées aux maladies de système : la sarcoïdose $(10,5 \%$ des cas), la maladie de Behçet (13,9\%) et la maladie de Vogt-Koyanagi-Hadara (5,9\%) ${ }^{5,14}$ (Figure 4).

\section{Uvéite et rhumatismes inflammatoires}

L'uvéite liée à l'antigène HLAB27 est l'une des causes les plus fréquentes d'uvéite antérieure. Elle peut être associée ou non à une spondyloarthrite. HLAB27 est présent chez $8 \%$ de la population caucasienne et chez plus de $80 \%$ des spondyloarthrites. Au cours de la spondylarthrite ankylosante, une uvéite antérieure aiguë se voit dans 20 à $30 \%$ des cas. Dans le syndrome de Fiessinger-Leroy qui associe inflammation oculaire, urétrite et arthrites, l'uvéite se voit dans 12 à $37 \%$ des cas, alors qu'elle est présente dans 7 à $25 \%$ des rhumatismes psoriasiques, dans 2 à $9 \%$ des maladies inflammatoires chroniques de l'intestin $(\mathrm{MICl})$ et dans $30 \%$ des arthrites indifférenciées ${ }^{16-18}$. 
Ces uvéites sont antérieures et touchent le plus souvent l'homme jeune (30-35 ans) avec un sex ratio $H / F$ de 1,5-2. La présentation clinique est caractérisée par un début brutal, des douleurs souvent intenses, une rougeur, une photophobie et une baisse de la vision modérée à sévère. L'atteinte est habituellement bilatérale, ou unilatérale à bascule, synéchiante, et surtout, non granulomateuse et non hypertensive ${ }^{17}$. La réaction inflammatoire peut être sévère avec la présence d'un hypopion dans $14 \%$ des cas.

Dans le cas particulier de la maladie de Crohn, l'uvéite, lorsqu'elle survient sur un terrain HLAB27, ne se différencie en rien des formes décrites aux cours des spondylo-arthrites. Cependant, des uvéites antérieures, granulomateuses et récidivantes ont également été décrites mais plus rares. La fréquence des récurrences est importante. Ces uvéites peuvent se compliquer de glaucome, de cataracte et d'hypertonie oculaire. Les complications du segment postérieur tel que l'œdème maculaire cystoïde peuvent être responsables d'une baisse de l'acuité visuelle et se voient dans $17 \%$ des $\operatorname{cas}^{17,18}$.

Au cours de la polyarthrite rhumatoïde, il n'a pas été décrit d'uvéite. Parmi les manifestations ophtalmologiques, on retrouve plutôt des sclérites et épisclérites ou des syndromes secs avec kératite ponctuée superficielle.

\section{Rappels sur les DMARDS et les biothérapies}

\section{- DMARDS}

Les DMARDS actuellement utilisés dans la prise en charge des maladies inflammatoires chroniques sont le méthotrexate (MTX), le leflunomide, la salazopyrine, les antipaludéens de synthèse type hydroxychloroquine et chloroquine, et l'azathioprine. Ces traitements permettent une épargne cortisonique et un bon contrôle de l'inflammation. Cependant, leurs effets immunomodulateurs et immunosuppresseurs demandent une attention particulière, notamment sur 
le plan infectieux. Par ailleurs, aucun lien direct n'a été mis en évidence entre ces traitements et la survenue d'uvéites.

- biothérapies

Depuis une quinzaine d'années, les biothérapies ont révolutionné la prise en charge des maladies inflammatoires rhumatismales, digestives et dermatologiques. Les anti-TNFa (adalimumab, infliximab, étanercept, golimumab, certolizumab) sont des molécules dirigées contre le TNFa, acteur central de la réaction inflammatoire. Le TNFa participe à l'activation du système immunitaire lors des processus infectieux ou néoplasiques mais possède un rôle délétère dans les processus inflammatoires chroniques. Les anticorps monoclonaux (Infliximab, adalimumab, golimumab) neutralisent le TNF après sa production avec une activité cytolytique, alors que les récepteurs solubles (étanercept) bloquent les cibles et que le certolizumab, fragment Fab d'lgG1, est un anticorps antagoniste.

L'anakinra est un inhibiteur de l'interleukine 1 (anti IL1) qui agit directement sur le récepteur en bloquant la voie de l'IL1, cytokine clé de l'inflammation et de l'immunité innée. Le tocilizumab est quant à lui un anticorps monoclonal humanisé anti lg G1 dirigé contre les récepteurs solubles et membranaires de l'IL6, cytokine pro-inflammatoire.

L'abatacept est une protéine de fusion entre l'antigène CTLA4 et le fragment Fc des IgG1. Elle possède une activité anti-inflammatoire par inhibition des réponses cellulaires $T$.

\section{Matériel et méthodes}

Par l'intermédiaire du Dr Bernadette Baldin, médecin de pharmacovigilance au CHU de Nice, nous avons interrogé la Base de Données Nationale de Pharmacovigilance et recherché les déclarations d'uvéites chez les patients traités par DMARDS et/ou biothérapies dans le cadre d'une maladie inflammatoire rhumatologique, digestive ou d'une connectivite, entre 2000 à 2015 en France. Tous les types d'uvéites ont été inclus. La recherche a été effectuée en tenant compte 
de la présentation anatomique : uvéites antérieures, intermédiaires, postérieures et panuvéites. Les DMARDS comprenaient le méthotrexate, le leflunomide, la sulfasalazine, les anti-malariques et l'azathioprine. Les biothérapies comprenaient tous les antiTNF alpha (étanercept, infliximab, adalimumab, golimumab et certolizumab) les anti-IL1 (anakinra) et IL6 (tocilizumab) mais aussi le rituximab (anti CD20) et l'abatacept (anti CTLA4). Les patients devaient être âgés de plus de 18 ans et présenter un rhumatisme inflammatoire tel que polyarthrite rhumatoïde (PR), spondyloarthrite (SPA) isolée ou associée à un psoriasis, maladie intestinale chronique inflammatoire (MICl) ou une connectivite.

\section{Résultats}

32 cas ont été rapportés, correspondant à 42 yeux atteints, dont 15 hommes (45\%) et 17 femmes (55\%). L'âge moyen était de 44 ans (19 - 70).

Dans cette population, il y avait :

- 14 SPA dont 13 spondylarthrites ankylosantes (SA) et 1 rhumatisme psoriasique (RP),

- 9 PR (dont une séronégative),

- 1 rhumatisme goutteux,

- $\quad 3 \mathrm{MICl}$,

- 1 psoriasis,

- 1 lupus,

- 3 rhumatismes inflammatoires inclassés.

2 patients avaient un DMARD seul (6\%): 1 était sous hydroxychloroquine et 1 sous méthotrexate. 30 patients sur 32 étaient sous biothérapie (94\%) dont 6 en association avec un DMARD. 17 d'entre eux étaient sous étanercept (53\%), 6 sous infliximab (19\%), 2 sous adalimumab (6\%) et 1 sous golimumab (3\%). 3 patients étaient sous tocilizumab (9\%), 1 sous anakinra (3\%). Aucun patient n'était sous abatacept, certolizumab ou rituximab (Table II). 
Concernant l'atteinte ophtalmologique, nous avons retrouvé :

- 19 uvéites antérieures (70.4\%), soit 27 yeux atteints, dont 11 UA unilatérales, (dont 1 uvéite hypertensive, 2 uvéites synéchiantes et non granulomateuses) et 8 UA bilatérales (dont 3 étaient synéchiantes, 2 hypertensives : 1 granulomateuse et 1 avec hypopion).

- 1 uvéite intermédiaire unilatérale $(3,7 \%)$.

- 5 uvéites postérieures (18,5\%), soit 7 yeux atteints : 3 UP unilatérales (dont 1 infectieuse herpétique chez une PR sous méthotrexate et tocilizumab), et 2 UP bilatérales d'étiologie infectieuse : 1 tuberculose chez une SPA sous étanercept, et 1 toxoplasmose chez un goutteux diabétique sous anakinra, corticoïdes et MTX.

- 2 panuvéites infectieuses (7,4\%): 1 toxoplasmose chez une PR sous infliximab et 1 uvéite herpétique chez une PR diabétique sous étanercept, MTX et corticoïdes.

- 5 déclarations ne permettaient pas de conclure car les uvéites étaient mal définies (Figure 5).

Les maladies sous-jacentes et les thérapeutiques en cours au moment de l'uvéite sont résumées dans la table III. Sur les 32 déclarations, il n'est jamais précisé si la maladie rhumatismale est active au plan articulaire par ailleurs au moment de l'uvéite.

Dans l'évolution, 9 patients ont vu leur traitement remplacé par une autre biothérapie (table III). Chez 8 autres, le traitement a été stoppé : 5 étanercept, 2 tocilizumab et 1 golimumab. Chez 5 patients, la biothérapie a été suspendue puis reprise après résolution de l'épisode oculaire. Le traitement a été poursuivi à l'identique sans interruption chez 4 patients (1 SPA sous adalimumab et 2 sous étanercept, 1 RP sous infliximab). Dans 6 cas, les données n'étaient pas disponibles.

\section{Discussion}

Ce travail nous a permis de mettre en évidence la survenue au cours de maladies inflammatoires chroniques traitées par biothérapies et/ou DMARDs d'uvéites de tout type anatomique et d'étiologies variées, incluant les atteintes spécifiques à la maladie et les causes infectieuses.

Sur les 32 uvéites déclarées, seuls 27 cas étaient complètement analysables. 
La population de ce registre correspond dans sa présentation globale aux caractéristiques des uvéites rapportées dans la littérature: l'âge moyen est comparable et le sex-ratio est de 0,83, proche du $1 / 1$ retrouvé dans la population uvéitique tout venant ${ }^{7}$. La proportion des uvéites antérieures de notre population est comparable à la littérature (respectivement $66.7 \%$ versus 66,5\%). Par contre, 8 des 27 uvéites n'étaient pas des uvéites antérieures : il s'agissait d'uvéites intermédiaires, postérieures ou de panuvéites (1 uvéite intermédiaire et 5 uvéites postérieures). Ces trois types d'uvéite, de fait, ne peuvent pas correspondre à des uvéites rhumatismales qui sont des uvéites antérieures, synéchiantes, uni ou bilatérales et ne sont jamais granulomateuses $^{17}$. De plus, les panuvéites, jamais attribuables à la maladie rhumatismale, représentaient $7,4 \%$ de notre cohorte ; elles représentent $15 \%$ des formes d'uvéites tout venant ${ }^{19}$. A noter, 5 de ces uvéites ont formellement été étiquetées infectieuses : une tuberculose, deux toxoplasmoses oculaires et deux uvéites herpétiques. On peut dès lors se poser la question du rôle propre de la biothérapie et des DMARDS dans l'augmentation du risque infectieux.

Dans notre étude, un seul patient SPA a présenté une authentique tuberculose pulmonaire et oculaire sous forme d'une uvéite postérieure bilatérale avec vascularite au fond d'œil. II s'agissait d'une primo-infection tuberculeuse, sous la forme d'une miliaire avec atteinte ophtalmique, alors que I'IDR était négative à l'introduction de l'étanercept; la rareté de cette complication sous étanercept nous a permis de publier cette observation ${ }^{20}$. Globalement, l'incidence des uvéites tuberculeuses va de 0,39 à $18 \%$ dans la littérature ${ }^{21}$. Elles sont de 2 types : tuberculose maladie par présence du bacille Mycobacterium tuberculosis dans les prélèvements oculaires, et tuberculose latente par réaction immunologique médiée, à distance d'un foyer tuberculeux méconnu ou mal traité ${ }^{21}$. Le risque de développer une atteinte oculaire est favorisé par l'immunosuppression liée aux corticostéroïdes et aux autres traitements agissant sur l'immunité ${ }^{21-}$ 23.

De nombreuses études montrent que le risque infectieux, notamment la tuberculose, est augmenté chez les patients atteints de rhumatismes inflammatoires, indépendamment de tout traitement par biothérapies. Les facteurs de risques majeurs identifiés sont : la corticothérapie associée ( $R R=2,2$ 
$[1,5-3,4])$, le tabagisme actif $(R R=1,6[1-2,5])$ et la positivité du facteur rhumatoïde $(R R=2[1,3-3])$ 19,25,26. Pour la moitié des cas dépistés, la réactivation d'une tuberculose latente sous antiTNFa survient dans la première année avec une proportion de $60 \%$ de tuberculoses extra-pulmonaires, contre $80 \%$ de formes pulmonaires pures dans la population générale ${ }^{26}$.

Deux toxoplasmoses oculaires ont été déclarées sous forme d'une uvéite postérieure bilatérale et d'une panuvéite unilatérale. Dans la population tout venant, la toxoplasmose oculaire est la première cause d'inflammation du segment postérieur en Europe $e^{5-7}$. Elle se caractérise par un foyer de rétinochoroïdite au fond d'œil se présentant sous forme d'une lésion blanchâtre, profonde, à bords flous, fréquemment satellite d'une lésion cicatricielle pigmentée et/ou atrophique. Le diagnostic se fait sur l'aspect du fond d'œil et peut être confirmé par PCR de l'humeur aqueuse lors d'une ponction de chambre antérieure ${ }^{15}$.

En cas d'immunodépression, on peut observer des toxoplasmoses oculaires plus sévères ou récidivantes : atteinte multifocale d'un seul œil, inflammation sévère bilatérale ou encore zone de nécrose rétinienne extensive. Ces atteintes peuvent être vues chez le patient $\mathrm{VIH}$ mais également dans les populations traitées par cortisone au long cours et/ou immunosuppresseurs ${ }^{27-30}$.

Deux uvéites herpétiques ont été prouvées dans notre étude. II s'agissait d'une uvéite postérieure et d'une panuvéite. Les infections virales du groupe herpès sont l'étiologie la plus fréquente des uvéites. II s'agit le plus souvent d'une uvéite antérieure, granulomateuse et hypertensive, pouvant s'associer à des manifestations extra-oculaires telles qu'un herpès labial ou une gingivo-stomatite. Le profil sérologique est peu contributif. La ponction de chambre antérieure avec analyse par PCR de l'humeur aqueuse peut permettre d'obtenir un diagnostic étiologique ${ }^{31-33}$. Dans notre groupe de patients, la fréquence des uvéites antérieures est très proche de celle de la population générale ${ }^{10-}$ 13. Or, l'origine rhumatismale de l'uvéite est supposée d'emblée à chaque fois, sauf dans 5 cas. Dans ce registre déclaratif, la description clinique ophtalmologique n'est pas suffisamment précise dans la majorité des cas pour une interprétation fine des données. Le caractère granulomateux de l'uvéite est indispensable pour le diagnostic car il exclut l'étiologie rhumatismale liée au HLA B27 
et oriente soit vers une étiologie infectieuse: virus du groupe herpès (Herpès simplex, CMV, HHV6...) le plus fréquemment, soit vers des granulomatoses telles que la tuberculose, la syphilis ou la sarcoïdose ${ }^{16-18}$. Dans notre étude, aucune donnée anatomique ne précise s'il s'agit d'uvéite synéchiante, qui pourrait correspondre effectivement à une atteinte spécifique, ou s'il s'agit d'une uvéite granulomateuse. Ces données manquantes, inhérentes à ce type de registre déclaratif, constitue une limite à l'interprétation des données. Ceci nous amène à nous demander quelle est la part rhumatismale spécifique et la part infectieuse parmi ces 22 uvéites qui n'ont pas eu de diagnostic étiologique formel. Enfin, l'activité inflammatoire articulaire du rhumatisme sous-jacent n'étant pas précisée, il est difficile de savoir si le patient était en phase d'échappement thérapeutique au moment de l'uvéite.

Les maladies inflammatoires, et notamment la polyarthrite rhumatoïde, sont reconnues comme facteur de risque infectieux en soi, mais les patients sont exposés à un risque supplémentaire lorsqu'ils sont sous agents cytotoxiques qui peuvent induire des neutropénies favorisant indirectement les phénomènes infectieux bactériens, viraux et fungiques ${ }^{34-36}$. Les études tirées du registre CORRONA ont montré une augmentation du risque infectieux sous MTX et sous antiTNFa par rapport aux autres DMARDS ${ }^{36}$. Par contre, il n'y avait pas d'effet cumulatif de l'association MTX et antiTNFa. II s'agissait principalement d'infections à germes communautaires touchant les voies aériennes supérieures ou d'infections virales à VZV'31,32,36,

Le risque infectieux est plus important sous traitements biologiques : infections à germes banals et candidoses mais également la tuberculose ${ }^{36-44}$. En effet, le risque de réactivation tuberculeuse de 2 à 4 fois plus important chez la population sous antiTNFa, avec un sur-risque pour les anticorps monoclonaux ${ }^{35}$

Dans les autres registres de Rhumatologie, le risque viral, notamment l'herpès et le zona, est augmenté quelle que soit la biothérapie et quelle que soit la population étudiée : PR, SPA, AJI ${ }^{44}$. Dans la population générale, les uvéites d'étiologie infectieuse prédominent, herpès principalement. Nous émettons donc l'hypothèse que la plus grande part des uvéites antérieures 
déclarées sous biothérapie est en fait de mécanisme infectieux, conséquence de l'immunodépression.

Enfin, concernant la thérapeutique, les uvéites n'ont jamais été décrites dans les essais thérapeutiques comme effets secondaires directs des biothérapies ou des DMARDS. Par contre, dans la littérature, il a été évoqué un lien entre le traitement par étanercept et la survenue d'uvéites, lors d'une communication à la Société Française de Rhumatologie (SFR) en 2010, repris en $2014^{45}$. Cet article suggérait une majoration du risque d'uvéite dans la première année de traitement par étanercept versus adalimumab, mais ce risque demeurait identique pour les autres biothérapies $^{45}$. Un lien entre étanercept et sclérite a également été évoqué ${ }^{46}$. Ces hypothèses n'ont jamais été confirmées et d'autres études ont montré depuis que l'incidence des uvéites n'était pas plus importante sous étanercept que sous une autre biothérapie ${ }^{47-49}$.

Enfin, les biothérapies et en particulier les anti TNFa sont actuellement utilisées dans le traitement des uvéites postérieures non infectieuses, notamment la maladie de Behçet, avec une RBU (Référentiels de Bon Usage hors GHS) pour infliximab, étanercept et adalimumab ${ }^{50-53}$.

\section{CONCLUSION}

Les uvéites peuvent être des manifestations propres des rhumatismes inflammatoires mais peuvent également apparaitre sous traitement, quelle que soit le type de biothérapie.

Les biothérapies avec ou sans DMARDS augmentent le risque infectieux, notamment viral.

Ainsi, la présence d'une uvéite sous traitement ne doit pas faire seulement évoquer un échappement thérapeutique, surtout si la manifestation oculaire est isolée. L'origine infectieuse ainsi favorisée doit être discutée systématiquement. L'identification de l'étiologie d'une uvéite survenant sous biothérapie est cruciale pour le pronostic visuel du patient et nécessite une prise en charge multidisciplinaire. 


\section{ANNEXES}

Table I - Principaux critères de classification des uvéites

Classification selon le terrain

Classification selon l'étiologie

Classification selon l'examen ophtalmologique
Âge du patient Infection et/ou inflammation

Maladie systémique ou uvéite isolée

Localisation

Mode évolutif

Intensité

Caractère " granulomateux "

plications anatomiques Complications fonctionnelles
Uvéites de l'enfant, de l'adulte ou du sujet âgé

Antérieure, intermédiaire, postérieure, panuvéite Uni- ou bilatérale (concomitante ou " à bascule ») Aigu ou chronique Chambre antérieure : tyndall cellulaire et flare Vitré : haze et cellularité vitréenne Présentation des précipités rétrodescemétiques Autres éléments " granulomateux » Synéchies, hypertonie, œdème papillaire \pm maculaire Retentissement de l'uvéite sur la vision

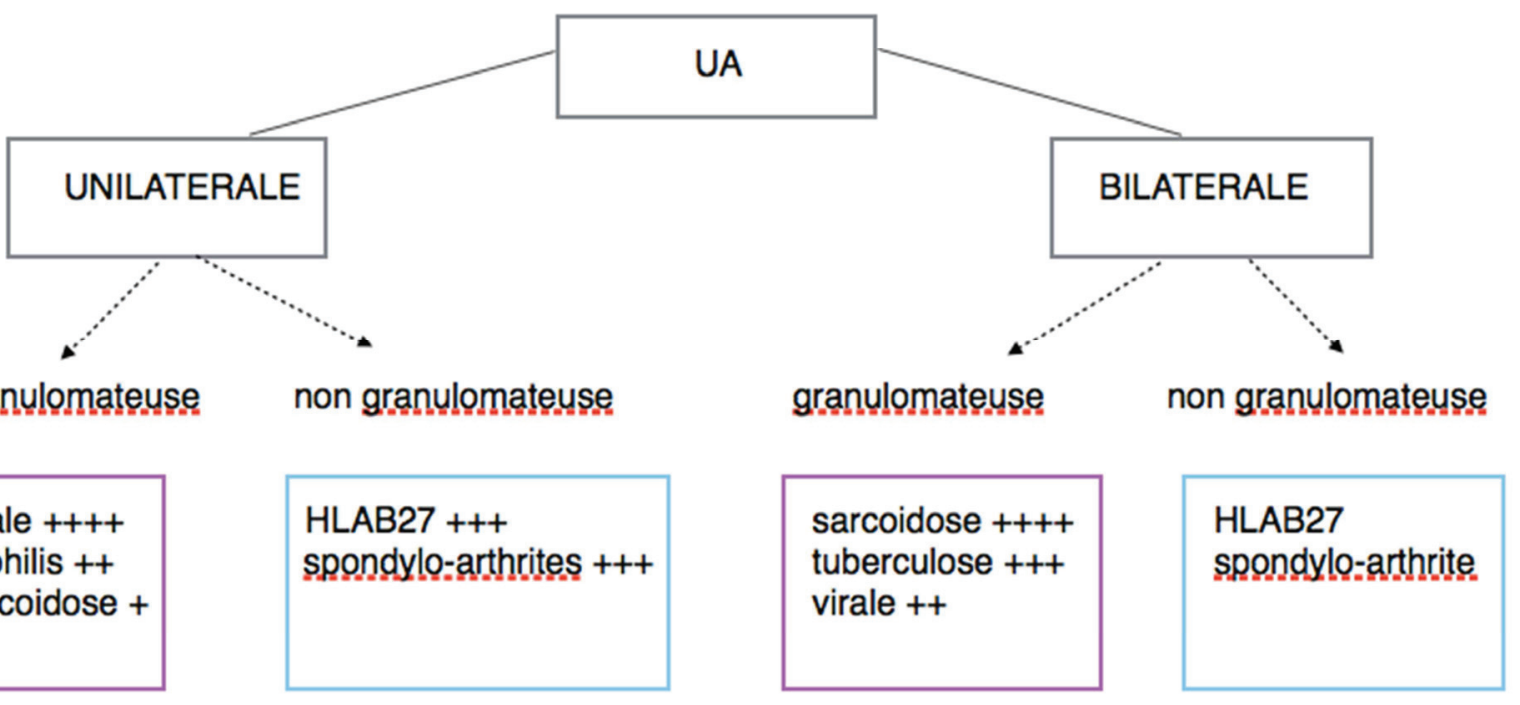

Schéma 1 - Classification des uvéites selon Bodaghi

UA : uvéite antérieure 
Fig 1 - Étiologies des UA

Fig 2 - Etiologies des UI

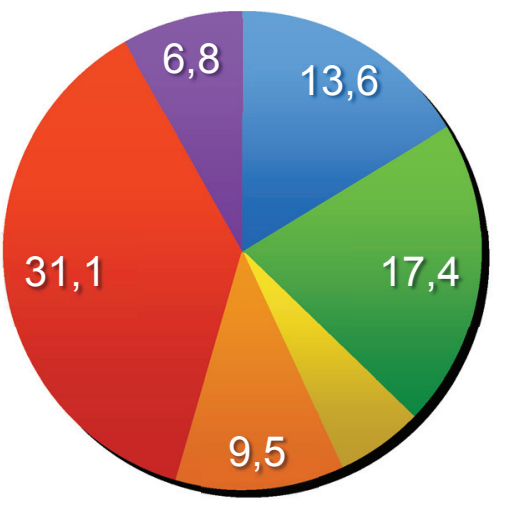

- idiopathique

- liées à HLAB27

- tuberculose

- cyclite de fuchs

- HSV/VZV

usarcoidose

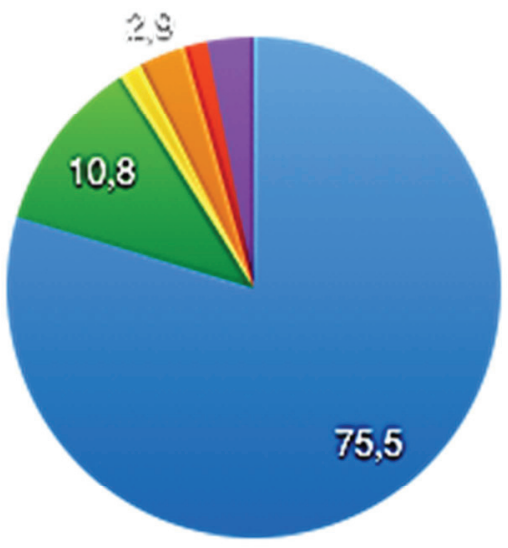

Fig 4 - Etiologies des panuvéites
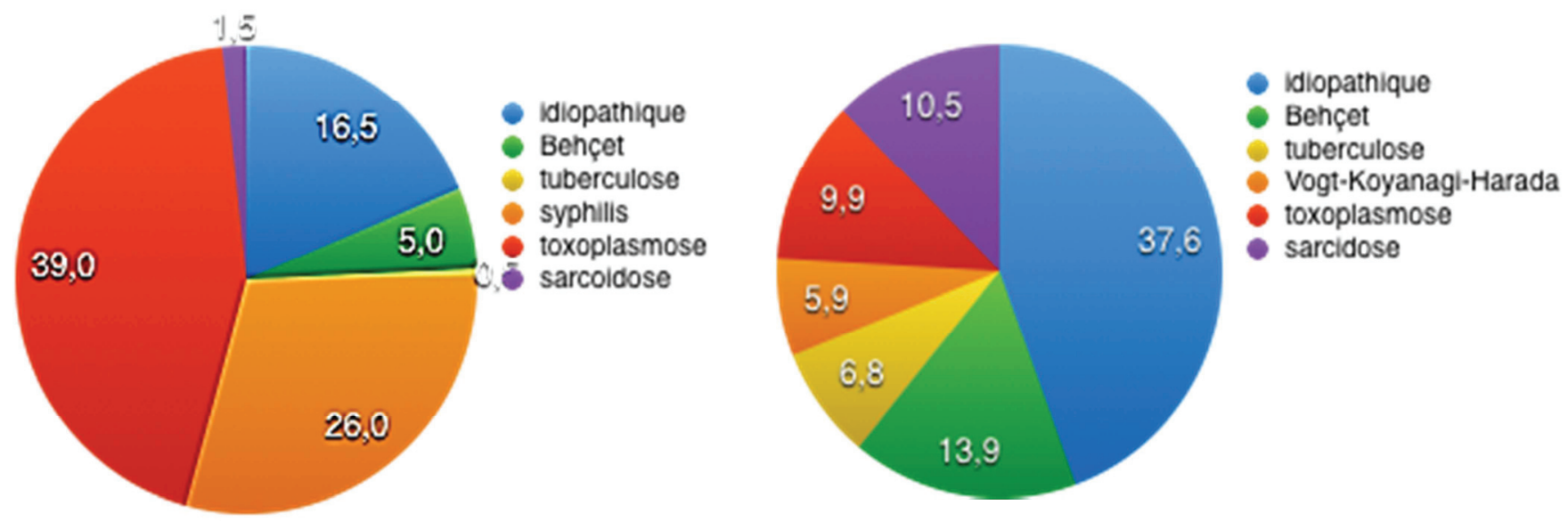

Fig 5 - Répartition des uvéites déclarées à la pharmacovigilance
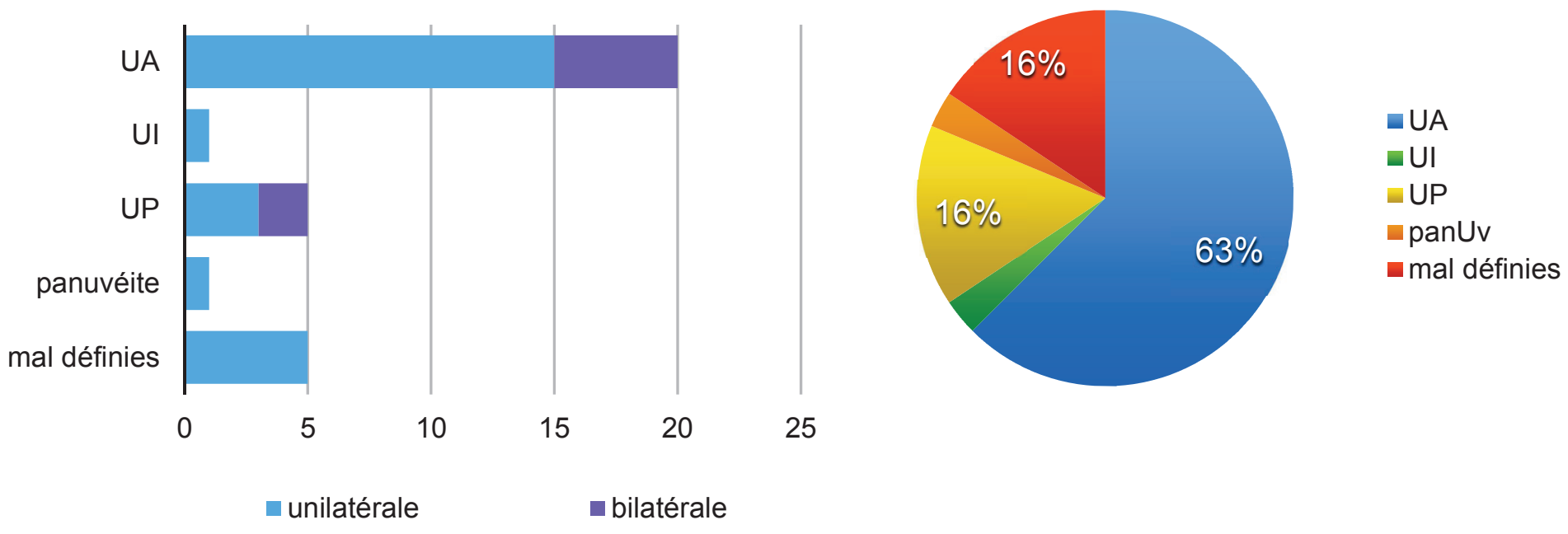
Table II - Caractéristiques des patients

\begin{tabular}{|c|c|c|c|c|c|}
\hline & Sexe & Age & Antécédents & maladie sous-jacente & traitement \\
\hline 1 & M & $43 \mathrm{~A}$ & $\begin{array}{l}\text { Dyslipidémie } \\
\text { Hypertension artérielle }\end{array}$ & $\begin{array}{l}\text { Spondylarthrite } \\
\text { ankylosante }\end{array}$ & étanercept 50 \\
\hline 2 & $\mathrm{~F}$ & $46 \mathrm{~A}$ & $\begin{array}{l}\text { Cancer du poumon } \\
\text { métastatique }\end{array}$ & Syndrome lupique & PLAQUENIL 200 2x/j \\
\hline 3 & M & $30 \mathrm{~A}$ & Spondylarthrite ankylosante & $\begin{array}{l}\text { Spondylarthrite } \\
\text { ankylosante }\end{array}$ & Tocilizumab \\
\hline 4 & $F$ & $49 \mathrm{~A}$ & Polyarthrite rhumatoïde & $\begin{array}{l}\text { Polyarthrite } \\
\text { rhumatoïde }\end{array}$ & $\begin{array}{l}\text { étanercept } 50 \text {, leflunomide } \\
20 \mathrm{mg} / \mathrm{j}\end{array}$ \\
\hline 5 & M & $60 \mathrm{~A}$ & HTA, dyslipidémie, IDM stenté & $\begin{array}{l}\text { Spondylarthrite } \\
\text { ankylosante }\end{array}$ & adalimumab 40 \\
\hline 6 & $F$ & $38 \mathrm{~A}$ & Cholécystectomie, Colectomie & $\begin{array}{l}\text { rhumatisme } \\
\text { inflammatoire inclassé }\end{array}$ & infliximab \\
\hline 7 & M & $51 \mathrm{~A}$ & & $\begin{array}{l}\text { rhumatisme } \\
\text { inflammatoire inclassé }\end{array}$ & étanercept 50 \\
\hline 8 & $F$ & $52 \mathrm{~A}$ & $\begin{array}{l}\text { Spondylarthrite } \\
\text { ankylosante }\end{array}$ & $\begin{array}{l}\text { Spondylarthrite } \\
\text { ankylosante }\end{array}$ & étanercept 50 \\
\hline 9 & M & $60 \mathrm{~A}$ & $\begin{array}{l}\text { Hypertension artérielle } \\
\text { Hyperuricémie, insuffisance } \\
\text { rénale }\end{array}$ & $\begin{array}{l}\text { Spondylarthrite } \\
\text { ankylosante }\end{array}$ & golimumab 50 \\
\hline 10 & M & $45 \mathrm{~A}$ & & Psoriasis & étanercept 50 \\
\hline 11 & $F$ & $59 \mathrm{~A}$ & Spondylarthrite ankylosante & $\begin{array}{l}\text { Spondylarthrite } \\
\text { ankylosante }\end{array}$ & infliximab \\
\hline 12 & $\mathrm{~F}$ & $31 \mathrm{~A}$ & Hypertension artérielle & Maladie de Crohn & adalimumab 40 \\
\hline 13 & M & $30 \mathrm{~A}$ & Hypertension artérielle & $\begin{array}{l}\text { rhumatisme } \\
\text { psoriasique }\end{array}$ & infliximab \\
\hline 14 & $F$ & $61 \mathrm{~A}$ & Diabète sucré de type II & $\begin{array}{l}\text { Polyarthrite } \\
\text { rhumatoïde }\end{array}$ & étanercept 50, MTX, СTO \\
\hline 15 & $F$ & $37 \mathrm{~A}$ & Spondylarthrite ankylosante & $\begin{array}{l}\text { Spondylarthrite } \\
\text { ankylosante }\end{array}$ & étanercept 50 \\
\hline 16 & $M$ & $20 \mathrm{~A}$ & $\mathrm{MICl}$ & $\mathrm{RCH}$ Corticorésistante & infliximab \\
\hline 17 & $\mathrm{~F}$ & $53 \mathrm{~A}$ & Polyarthrite rhumatoïde & Polyarthrite rhumatoïde & étanercept 50 \\
\hline
\end{tabular}


Table II - Caractéristiques des patients

\begin{tabular}{|c|c|c|c|c|c|}
\hline & Sexe & Age & Antécédents & maladie sous-jacente & traitement \\
\hline 18 & $F$ & NA & Polyarthrite rhumatoïde & $\begin{array}{l}\text { Polyarthrite } \\
\text { rhumatoïde }\end{array}$ & étanercept 50 \\
\hline 19 & $F$ & $31 \mathrm{~A}$ & $\mathrm{MICl}$ & Maladie de Crohn & infliximab 340 \\
\hline 20 & $M$ & $36 \mathrm{~A}$ & Spondylarthrite ankylosante & $\begin{array}{l}\text { Spondylarthrite } \\
\text { ankylosante }\end{array}$ & étanercept 25 2x/s \\
\hline 21 & $F$ & $46 \mathrm{~A}$ & Polyarthrite rhumatoïde & $\begin{array}{l}\text { Polyarthrite } \\
\text { rhumatoïde neg }\end{array}$ & étanercept 25 \\
\hline 22 & $M$ & $19 \mathrm{~A}$ & Spondylarthrite ankylosante & $\begin{array}{l}\text { Spondylarthrite } \\
\text { ankylosante }\end{array}$ & $\begin{array}{l}\text { étanercept } 25 \text {, } \\
\text { sulfasalazine } 5003 x / j\end{array}$ \\
\hline 23 & $\mathrm{~F}$ & $47 \mathrm{~A}$ & $\begin{array}{l}\text { rhumatisme inflammatoire } \\
\text { inclassé }\end{array}$ & $\begin{array}{l}\text { rhumatisme } \\
\text { inflammatoire inclassé }\end{array}$ & $\begin{array}{l}\text { tocilizumab, MTX } 20, \text { СТО } \\
5 \mathrm{mg} / \mathrm{j}\end{array}$ \\
\hline 24 & $\mathrm{~F}$ & $67 \mathrm{~A}$ & Allergie médicamenteuse & $\begin{array}{l}\text { Polyarthrite } \\
\text { rhumatoïde }\end{array}$ & MTX 20, СТО 10mg/j \\
\hline 25 & $M$ & $39 \mathrm{~A}$ & Spondylarthrite ankylosante & $\begin{array}{l}\text { Spondylarthrite } \\
\text { ankylosante }\end{array}$ & étanercept 50 \\
\hline 26 & $M$ & $39 \mathrm{~A}$ & Spondylarthrite ankylosante & $\begin{array}{l}\text { Spondylarthrite } \\
\text { ankylosante }\end{array}$ & étanercept 50 \\
\hline 27 & $M$ & $28 \mathrm{~A}$ & Spondylarthrite ankylosante & $\begin{array}{l}\text { Spondylarthrite } \\
\text { ankylosante }\end{array}$ & étanercept 50 \\
\hline 28 & $F$ & $32 \mathrm{~A}$ & Polyarthrite rhumatoïde & $\begin{array}{l}\text { Polyarthrite } \\
\text { rhumatoïde }\end{array}$ & $\begin{array}{l}\text { étanercept } 50 \text {, leflunomide } \\
20, \text { CTO } 4 \mathrm{mg} / \mathrm{j}\end{array}$ \\
\hline 29 & $F$ & $65 \mathrm{~A}$ & Polyarthrite rhumatoïde & $\begin{array}{l}\text { Polyarthrite } \\
\text { rhumatoïde }\end{array}$ & infliximab \\
\hline 30 & $F$ & $54 \mathrm{~A}$ & Polyarthrite rhumatoïde & Polyarthrite rhumatoïde & $\begin{array}{l}\text { tocilizumab, MTX } \\
20 \mathrm{mg} / \mathrm{sem}\end{array}$ \\
\hline 31 & $M$ & $24 \mathrm{~A}$ & Spondylarthrite ankylosante & $\begin{array}{l}\text { Spondylarthrite } \\
\text { ankylosante }\end{array}$ & étanercept 50 \\
\hline 32 & $M$ & $70 \mathrm{~A}$ & diabète, Insuffisance rénale & goutte polyarticulaire & Anakinra \\
\hline
\end{tabular}

$\mathrm{CO}=$ corticothérapie orale, $\mathrm{MTX}=$ méthotrexate 
Table III - Caractéristiques cliniques des uvéites

\begin{tabular}{|c|c|c|c|}
\hline & type d'uvéite & maladie sous jacente & $\begin{array}{l}\text { traitement en cours et } \\
\text { évolution du traitement }\end{array}$ \\
\hline 1 & UA OG, hypertensive & Spondylarthrite ankylosante & adalimumab $\rightarrow$ poursuite \\
\hline 2 & UA OD, oedème maculaire & Spondylarthrite ankylosante & golimumab $\rightarrow$ stop \\
\hline 3 & UA OG & Spondylarthrite ankylosante & étanercept $\rightarrow$ adalimumab \\
\hline 4 & UA OG & Spondylarthrite ankylosante & étanercept $\rightarrow$ adalimumab \\
\hline 5 & UA OG & Spondylarthrite ankylosante & étanercept $\rightarrow$ poursuite \\
\hline 6 & UA OG & Spondylarthrite ankylosante & étanercept $\rightarrow$ poursuite \\
\hline 7 & $\begin{array}{l}\text { UA OD, synéchies, non } \\
\text { granulomateuse }\end{array}$ & Spondylarthrite ankylosante & étanercept $\rightarrow$ adalimumab \\
\hline 8 & $\begin{array}{l}\text { UA OD, hypopion, synéchies, } \\
\text { non granulomateuse }\end{array}$ & Spondylarthrite ankylosante & étanercept + sulfasalazine \\
\hline 9 & UA OG, oedème papillaire & PR sérodéfinie & $\begin{array}{l}\text { tocilizumab + MTX 20mg } \\
\rightarrow \text { stop }\end{array}$ \\
\hline 10 & UA OG à œil blanc, chronique & PR non sérodéfinie & étanercept $\rightarrow$ stop \\
\hline 11 & UA OD & $\begin{array}{l}\text { Rhumatisme inflammatoire } \\
\text { inclassé }\end{array}$ & infliximab $\rightarrow$ suspendu \\
\hline 12 & UA bilatérale, synéchies & Spondylarthrite ankylosante & étanercept $\rightarrow$ infliximab \\
\hline 13 & $\begin{array}{l}\text { UA bilatérale, granulomateuse, } \\
\text { hypertensive, nodules de } \\
\text { Koeppe }\end{array}$ & PR sérodéfinie & étanercept $\rightarrow$ stop \\
\hline 14 & $\begin{array}{l}\text { UA bilatérale non } \\
\text { granulomateuse }\end{array}$ & PR sérodéfinie & $\begin{array}{l}\text { étanercept + leflunomide } \\
\rightarrow \text { certolizumab }\end{array}$ \\
\hline 15 & $\begin{array}{l}\text { UA bilatérale, non } \\
\text { granulomateuse, synéchies }\end{array}$ & PR sérodéfinie & $\begin{array}{l}\text { étanercept + leflunomide + } \\
\text { CTO } \rightarrow \text { stop }\end{array}$ \\
\hline 16 & UA bilatérale, synéchies & $\begin{array}{l}\text { Rhumatisme inflammatoire } \\
\text { inclassé }\end{array}$ & étanercept $\rightarrow$ leflunomide \\
\hline 17 & $\begin{array}{l}\text { UA bilatérale, oedème } \\
\text { maculaire }\end{array}$ & Psoriasis & étanercept $\rightarrow$ suspendu \\
\hline
\end{tabular}


UA bilatérale, hypertensive,

Maladie de Crohn

adalimumab hypopion

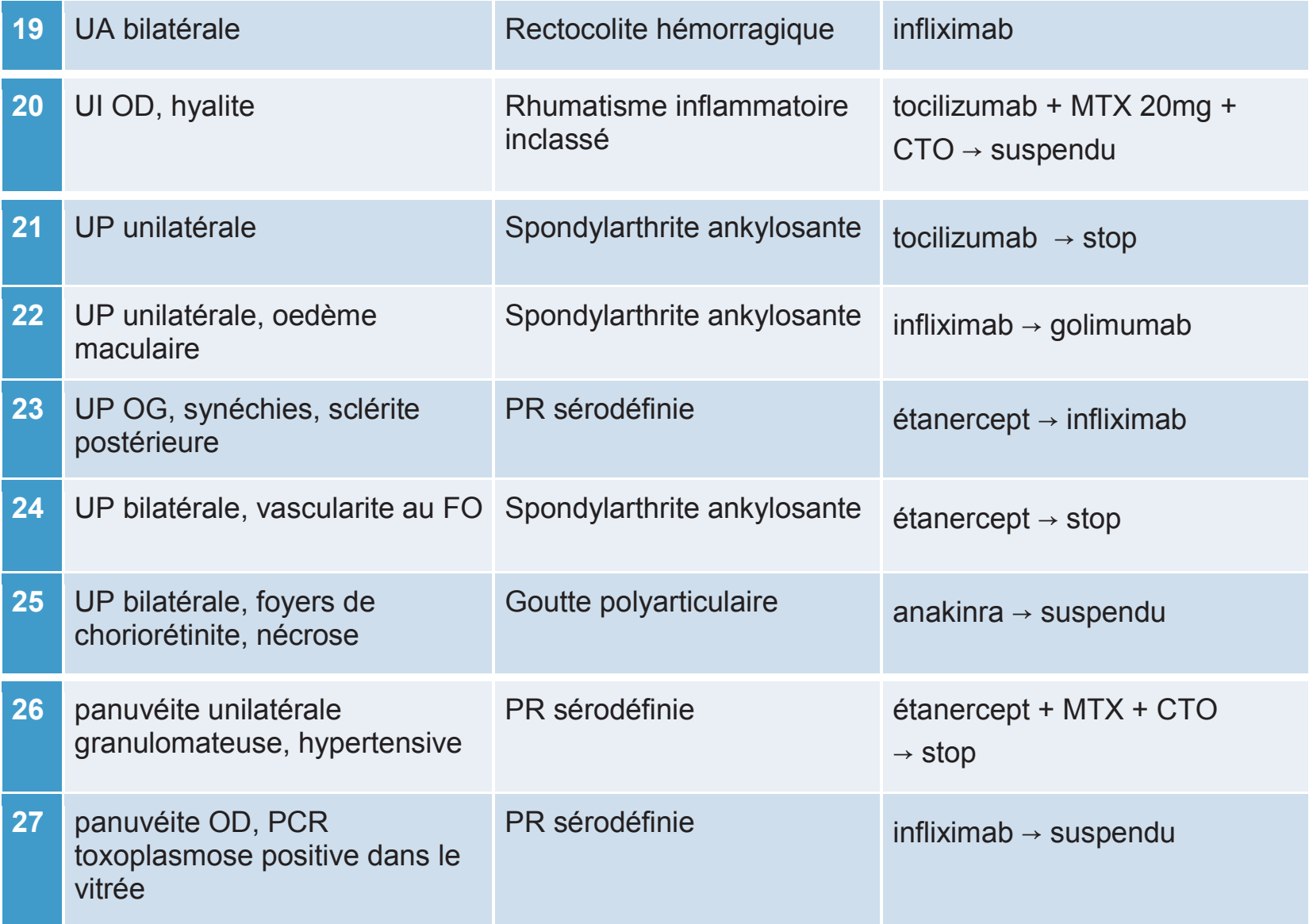

MTX : méthotrexate, CTO : corticothérapie orale

UA : uvéite antérieure, UI : uvéite intermédiaire, UP : uvéite postérieure

$\mathrm{PR}$ : polyarthrite rhumatoïde 


\section{Bibliographie}

1. Durrani OM, Tehrani NN, Marr JE, Moradi P, Stavrou P, Murray PI. Degree, duration, and causes of visual loss in uveitis. Br J Ophthalmol. 2004 Sep;88(9):1159-62.

2. Durrani OM, Meads CA, Murray PI. Uveitis: a potentially blinding disease. Ophthalmologica. 2004 Jul-Aug;218(4):223-36. Review.

3. Rosenbaum JT. Uveitis in spondyloarthritis including psoriatic arthritis, ankylosing spondylitis, and inflammatory bowel disease. Clin Rheumatol. 2015 Jun;34(6):999-1002. doi: $10.1007 / \mathrm{s} 10067-015-2960-8$.

4. Stolwijk C, van Tubergen A, Castillo-Ortiz JD, Boonen A. Prevalence of extra-articular manifestations in patients with ankylosing spondylitis: a systematic review and meta-analysis. Ann Rheum Dis. 2015 Jan;74(1):65-73.

5. Bodaghi B, Wechsler B, Du-Boutin LT, Cassoux N, LeHoang P, Piette JC. [Chronic severe uveitis: classification, search for etiology and therapeutic approach].Rev Med Interne. 2003 Dec;24(12):794-802.

6. Grajewski RS, Caramoy A, Frank KF, Rubbert-Roth A, Fätkenheuer G, Kirchhof B, Cursiefen C, Heindl LM. Spectrum of Uveitis in A German Tertiary Center: Review of 474 Consecutive Patients. Ocul Immunol Inflamm. 2015 Mar 11:1-7.

7. Jakob E, Reuland MS, Mackensen F, Harsch N, Fleckenstein M, Lorenz HM, Max R, Becker MD. Uveitis subtypes in a german interdisciplinary uveitis center--analysis of 1916 patients. J Rheumatol. 2009 Jan;36(1):127-36.

8. Nguyen AM, Sève P, Le Scanff J, Gambrelle J, Fleury J, Broussolle C, Grange JD, Kodjikian L. [Clinical and etiological aspects of uveitis: a retrospective study of 121 patients referred to a tertiary centre of ophthalmology]. Rev Med Interne. 2011 Jan;32(1):9-16.

9. Bloch-Michel E, Nussenblatt RB. International Uveitis Study Group recommendations for the evaluation of intraocular inflammatory disease. Am J Ophthalmol. 1987 Feb15;103(2):234-5.

10. Reeves SW, Sloan FA, Lee PP, Jaffe GJ. Uveitis in the elderly: epidemiological data from the National Long-term Care Survey Medicare Cohort. Ophthalmology. 2006 Feb;113(2):307.

11. Bajwa A, Osmanzada D, Osmanzada S, Khan I, Patrie J, Xin W, Reddy AK. Epidemiology of uveitis in the mid-Atlantic United States. Clinical Ophthalmology (Auckland, N.Z.). 1/01/01 00:00; 9: 889-901

12. Acharya NR, Tham VM, Esterberg E, Borkar DS, Parker JV, Vinoya AC, Uchida A. Incidence and prevalence of uveitis: results from the Pacific Ocular Inflammation Study. JAMA Ophthalmol. 2013 Nov;131(11):1405-12.

13. Gritz DC, Wong IG. Incidence and prevalence of uveitis in Northern California;the Northern California Epidemiology of Uveitis Study. Ophthalmology. 2004 Mar;111(3):491-500; 
14. McCannel CA, Holland GN, Helm CJ, Cornell PJ, Winston JV, Rimmer TG. Causes of uveitis in the general practice of ophthalmology. UCLA Community-Based Uveitis Study Group. Am J Ophthalmol. 1996 Jan;121(1):35-46.

15. Lin SJ, Lee SE, Kim SH, Hong SH, You YS, Kwon OW, Kim HS. Prevalence of Toxoplasma gondii and Toxocara canis among patients with uveitis. Ocul Immunol Inflamm. 2014 Oct;22(5):360-6.

16. Zarco P, González CM, Rodríguez de la Serna A, Peiró E, Mateo I, Linares L, Calvo J, CeaCalvo L, Arteaga MJ, Vanaclocha F, Marín-Jiménez I, García-Vicuña R. Extra-articular disease in patients with spondyloarthritis. Baseline characteristics of the spondyloarthritis cohort of the AQUILES study. Reumatol Clin. 2015 Mar-Apr;11(2):83-9.

17. Chang JH, McCluskey PJ, Wakefield D. Acute anterior uveitis and HLA-B27. Surv Ophthalmol. 2005 Jul-Aug;50(4):364-88. Review.

18. Monnet D, Breban M, Hudry C, Dougados M, Brézin AP. Ophthalmic findings and frequency of extraocular manifestations in patients with HLA-B27 uveitis: a study of 175 cases.

Ophthalmology. 2004 Apr;111(4):802-9.

19. Etude ULISSE : difficultés étiologiques dans les uvéites

20. Cabane L, Baillif S, Asrarguis G, Gastaud P, Queyrel V, Tieulié N. Uveitis associated with spondylitis: It may be tuberculosis!. J Fr Ophtalmol. 2016 Apr;39(4):e83-4

21. Gupta A, Sharma A, Bansal R, Sharma K. Classification of intraocular tuberculosis. Ocul Immunol Inflamm. 2015 Feb;23(1):7-13.

22. Gupta V, Shoughy SS, Mahajan S, Khairallah M, Rosenbaum JT, Curi A, Tabbara KF. Clinics of ocular tuberculosis. Ocul Immunol Inflamm. 2015 Feb;23(1):14-24.

23. Ponce de Leon D, Acevedo-Vasquez E, Alvizuri S, Gutierrez C, Cucho M, Alfaro J, Perich R, Sanchez-Torres A, Pastor C, Sanchez-Schwartz C, Medina M, Gamboa R, Ugarte M. Comparison of an interferon-gamma assay with tuberculin skin testing for detection of tuberculosis (TB) infection in patients with rheumatoid arthritis in a TB-endemic population. $J$ Rheumatol. 2008 May;35(5):776-81.

24. Ang M, Htoon HM, Chee SP. Diagnosis of tuberculous uveitis: clinical application of an interferon-gamma release assay. Ophthalmology. 2009 Jul;116(7):1391-6.

25. Van der Veen MJ, van der Heide A, Kruize AA, Bijlsma JW. Infection rate and use of antibiotics in patients with rheumatoid arthritis treated with methotrexate. Ann Rheum Dis. 1994 Apr;53(4):224-8.

26. Djebbar M. infection risk when receiving anti TNF treatment: crucial and major concern or not so bad reality? Rev Mar Rhum 2015; 31 : 34-44

27. Rodrigues KF, Faria e Arantes TE, Muccioli C, Neto JL, Pinheiro MM. Incidence of Toxoplasma retinochoroiditis in patients with ankylosing spondylitis after using TNF- $\alpha$ blockers. Parasitol Int. 2013 Jun;62(3):272-5.

28. Lassoued S, Zabraniecki L, Marin F, Billey T. Toxoplasmic chorioretinitis and antitumor necrosis factor treatment in rheumatoid arthritis. Semin Arthritis Rheum. 2007 Feb;36(4):262-3.

29. Smith JR, Cunningham ET Jr. Atypical presentations of ocular toxoplasmosis. Curr Opin Ophthalmol. 2002 Dec;13(6):387-92. Review. 
30. Nicholson $\mathrm{DH}$, Wolchok EB. Ocular toxoplasmosis in an adult receiving long-term corticosteroid therapy. Arch Ophthalmol. 1976 Feb;94(2):248-54.

31. Strangfeld A, Listing J, Herzer P, Liebhaber A, Rockwitz K, Richter C, Zink A. Risk of herpes zoster in patients with rheumatoid arthritis treated with anti-TNF-alpha agents. JAMA. 2009 Feb 18;301(7):737-44.

32. Serac G, Tubach F, Mariette X, Salmon-Céron D, Ravaud P, Lioté F, Laharie D, Ziza JM, Marguerie L, Bonnet C, Falgarone G, Nicolas N, Lortholary O, Chosidow O. Risk of herpes zoster in patients receiving anti-TNF- $\alpha$ in the prospective French RATIO registry. J Invest Dermatol. 2012 Mar;132(3 Pt 1):726-9.

33. Che H, Lukas C, Morel J, Combe B. Risk of herpes/herpes zoster during anti-tumor necrosis factor therapy in patients with rheumatoid arthritis. Systematic review and meta-analysis. Joint Bone Spine. 2014 May;81(3):215-21.

34. Franklin J, Lunt M, Bunn D, Symmons D, Silman A. Risk and predictors of infection leading to hospitalisation in a large primary-care-derived cohort of patients with inflammatory polyarthritis. Ann Rheum Dis. 2007 Mar;66(3):308-12.

35. Emery P, Gallo G, Boyd H, Morgan CL, Currie CJ, Poole CD, Nab HW. Association between disease activity and risk of serious infections in subjects with rheumatoid arthritis treated with etanercept or disease-modifying anti-rheumatic drugs. Clin Exp Rheumatol. 2014 SepOct;32(5):653-60. Epub 2014 Sep 5.

36. Greenberg JD, Reed G, Kremer JM, Tindall E, Kavanaugh A, Zheng C, Bishai W, Hochberg MC; CORRONA Investigators. Association of methotrexate and tumour necrosis factor antagonists with risk of infectious outcomes including opportunistic infections in the CORRONA registry. Ann Rheum Dis. 2010 Feb;69(2):380-6.

37. Haerter G, Manfras BJ, de Jong-Hesse Y, Wilts H, Mertens T, Kern P, Schmitt M. Cytomegalovirus retinitis in a patient treated with anti-tumor necrosis factor alpha antibody therapy for rheumatoid arthritis. Clin Infect Dis. 2004 Nov 1;39(9):e88-94.

38. Segal BH, Sneller MC. Infectious complications of immunosuppressive therapy in patients with rheumatic diseases. Rheum Dis Clin North Am. 1997 May;23(2):219-37. Review.

39. Mikuls TR, Moreland LW. Benefit-risk assessment of infliximab in the treatment of rheumatoid arthritis. Drug Saf. 2003;26(1):23-32. Review.

40. Wiland P, Głowska A, Chlebicki A, Szechiński J. [Analysis of efficacy and safety of multiple intravenous infusion of anti-tumor necrosis factor-alpha monoclonal antibody (Remicade) combined with methotrexate compared with sodium aurothiomalate and intramuscular depot methylprednisolone in rheumatoid arthritis]. Pol Arch Med Wewn. 2002 Nov;108(5):1055-63. Polish.

41. Bongartz T, Sutton AJ, Sweeting MJ, Buchan I, Matteson EL, Montori V. Anti-TNF antibody therapy in rheumatoid arthritis and the risk of serious infections and malignancies: systematic review and meta-analysis of rare harmful effects in randomized controlled trials. JAMA. 2006 Jun 7;295(21):2482.

42. Engelhard SB, Haddad Z, Bajwa A, Patrie J, Xin W, Reddy AK. Infectious uveitis in Virginia. Clin Ophthalmol. 2015;9:1589-94.

43. Lim WS, Powell RJ, Johnston ID. Tuberculosis and treatment with infliximab. N Engl J Med. 2002 Feb 21;346(8):623-6. 
44. Ruderman EM. Overview of safety of non-biologic and biologic DMARDs. Rheumatology (Oxford). 2012 Dec;51 Suppl 6:vi37-43.

45. Wendling $D^{1}$, Joshi A, Reilly $P$, Jalundhwala $Y J$, Mittal M, Bao Y. Comparing the risk of developing uveitis in patients initiating anti-tumor necrosis factor therapy for ankylosing spondylitis: an analysis of a large US claims database. Curr Med Res Opin. 2014 Dec;30(12):2515-21.

46. Gaujoux-Viala C, Giampietro C, Gaujoux T, Ea HK, Prati C, Orcel P, Wendling D, Lioté F. Scleritis: a paradoxical effect of etanercept? Etanercept-associated inflammatory eye disease. J Rheumatol. 2012 Apr;39(4):881.

47. Lim LL, Fraunfelder FW, Rosenbaum JT. Do tumor necrosis factor inhibitors cause uveitis? A registry-based study. Arthritis Rheum. 2007 Oct;56(10):3248-52.

48. Otten MH, Anink J, Prince FH, Twilt M, Vastert SJ, ten Cate R, Hoppenreijs EP, Armbrust W, Gorter SL, van Pelt PA, Kamphuis SS, Dolman KM, Swart JF, van den Berg JM, KoopmanKeemink Y, van Rossum MA, Wulffraat NM, van Suijlekom-Smit LW. Trends in prescription of biological agents and outcomes of juvenile idiopathic arthritis: results of the Dutch national Arthritis and Biologics in Children Register. Ann Rheum Dis. 2015 Jul;74(7):1379-86.

49. Kearsley-Fleet L, Davies R, Lunt M, Southwood TR, Hyrich KL. Factors associated with improvement in disease activity following initiation of etanercept in children and young people with Juvenile Idiopathic Arthritis: results from the British Society for Paediatric and Adolescent Rheumatology Etanercept Cohort Study. Rheumatology (Oxford). 2016 May;55(5):840-7

50. Feurer E, Bielefeld P, Saadoun D, Sève P. [Biologics in uveitis]. Rev Med Interne. 2015 Feb;36(2):107-16.

51. Abad S, Sève P, Dhote R, Brézin AP. [Guidelines for the management of uveitis in internal medicine]. Rev Med Interne. 2009 Jun;30(6):492-500.

52. Desbois AC, Addimanda O, Bertrand A, Deroux A, Pérard L, Depaz R, Hachulla E, Lambert M, Launay D, Subran B, Ackerman F, Mariette X, Cohen F, Marie I, Salvarini C, Cacoub P, Saadoun D. Efficacy of Anti-TNFa in Severe and Refractory Neuro-Behcet Disease: An Observational Study. Medicine (Baltimore). 2016 Jun;95(23):e3550

53. AFFSAPS - Référentiels de Bon Usage hors GHS AntiTNF. MAJ Novembre 2012 


\section{Introduction}

Face à la survenue d'une uvéite chez les patients traités par biothérapie et/ou DMARDs, le rhumatologue doit en préciser rapidement l'origine pour optimiser la prise en charge. L'uvéite est une inflammation intra-oculaire qui peut représenter une menace pour le pronostic visuel des patients. Les uvéites représentent $10 \%$ des causes de cécité dans les pays industrialisés. ${ }^{1,2}$

La nécessité d'un diagnostic précis chez un patient traité pour un rhumatisme inflammatoire chronique vient du fait que les uvéites peuvent être d'origine inflammatoire, en rapport avec la maladie sous-jacente (la spondylarthrite ankylosante est associée dans 5 à $15 \%$ des cas à des uvéites spécifiques $\left.{ }^{3,4}\right)$, mais également d'origine infectieuse, favorisée par l'immunodépression liée à la biothérapie et/ou aux DMARDs. A ce jour, nous ne disposons pas de données sur les étiologies des uvéites chez des patients traités par biothérapie et/ou DMARDs pour une maladie inflammatoire chronique ${ }^{5-8}$.

Dans la population générale, les uvéites font partie d'un groupe de maladies hétérogènes et complexes, représentant un véritable défi diagnostique et thérapeutique pour les praticiens. Les données épidémiologiques ont longtemps été rares et incomplètes en raison du manque de définition commune au terme uvéite et de ses différentes entités cliniques. L'International Uveitis Study Group a toutefois permis une standardisation des descriptions cliniques. ${ }^{9}$ (Table I). Les uvéites surviennent à tout âge avec un sex ratio de 1 de façon générale. Leur incidence est estimée à 17 à 32/100 000 habitants par an et leur prévalence serait de 38 à 204/100 000 habitants, ce qui correspond à une incidence de 9350-13 200 cas par an en France toutes causes confondues. ${ }^{10-13}$

Le but de notre travail a été :

- de décrire le type d'uvéites apparues chez des patients présentant un rhumatisme inflammatoire, une maladie chronique de l'intestin ( $\mathrm{MICl}$ ) ou dermatologique (psoriasis), traités par biothérapie et/ou DMARDS et ayant fait l'objet d'une déclaration à la pharmacovigilance 
- d'évaluer quelle proportion d'uvéites correspond à une poussée spécifique de la maladie, quelle proportion correspond à une complication infectieuse et si un effet secondaire direct du médicament peut être retenue comme étiologie.

\section{Matériel et méthodes}

Par l'intermédiaire du Dr Bernadette Baldin, médecin de pharmacovigilance au CHU de Nice, nous avons interrogé la Base de Données Nationale de Pharmacovigilance et recherché les déclarations d'uvéites chez les patients traités par DMARDS et/ou biothérapies dans le cadre d'une maladie inflammatoire rhumatologique, digestive ou d'une connectivite, entre 2000 à 2015 en France. Tous les types d'uvéites ont été inclus. La recherche a été effectuée en tenant compte de la présentation anatomique : uvéites antérieures (UA), intermédiaires (UI), postérieures (UP) et panuvéites(PANUv). Les DMARDS comprenaient le méthotrexate, le leflunomide, la sulfasalazine, les anti-malariques et l'azathioprine. Les biothérapies comprenaient tous les antiTNF alpha (etanercept, infliximab, adalimumab, golimumab et certolizumab) les anti-IL1 (anakinra) et IL6 (tocilizumab) mais aussi le rituximab (anti CD20) et l'abatacept (anti CTLA4). Les patients devaient être âgés de plus de 18 ans et présenter un rhumatisme inflammatoire tel que polyarthrite rhumatoïde (PR), spondylo-arthrite (SPA) isolée ou associée à un psoriasis, maladie intestinale chronique inflammatoire $(\mathrm{MICl})$ ou une connectivite.

\section{Résultats}

32 cas ont été rapportés, correspondant à 42 yeux atteints, dont 15 hommes (45\%) et 17 femmes (55\%). L'âge moyen était de 44 ans [19 - 70]. Dans cette population, il y avait : 14 SPA dont 13 spondylarthrites ankylosantes (SA) et 1 rhumatisme psoriasique (RP), 9 PR (dont une séronégative), 1 rhumatisme goutteux, $3 \mathrm{MICl}, 1$ psoriasis, 1 lupus, 3 rhumatismes inflammatoires inclassés.

2 patients avaient un DMARD seul (6\%) : 1 était sous hydroxychloroquine et 1 sous méthotrexate. 30 patients sur 32 étaient sous biothérapie (94\%) dont 6 en association avec un DMARD. 
17 d'entre eux (53\%) étaient sous etanercept, 6 sous infliximab (19\%), 2 sous adalimumab (6\%) et 1 sous golimumab (3\%). 3 patients étaient sous tocilizumab (9\%), 1 sous anakinra (3\%). Aucun patient n'était sous abatacept, certolizumab ou rituximab (table II).

Concernant l'atteinte ophtalmologique, nous avons retrouvé :

- 19 uvéites antérieures (70.4\%), soit 27 yeux atteints, dont 11 UA unilatérales, (dont 1 uvéite hypertensive, 2 uvéites synéchiantes et non granulomateuses) et 8 UA bilatérales (dont 3 étaient synéchiantes, 2 hypertensives : 1 granulomateuse et 1 avec hypopion).

- 1 uvéite intermédiaire unilatérale $(3,7 \%)$.

- 5 uvéites postérieures (18,5\%), soit sept yeux atteints : 3 UP unilatérales (dont 1 infectieuse herpétique chez une PR sous méthotrexate et tocilizumab), et 2 UP bilatérales d'étiologie infectieuse : 1 tuberculose chez une SPA sous etanercept, et 1 toxoplasmose chez un goutteux diabétique sous anakinra, corticoïdes et MTX.

- 2 panuvéites infectieuses (7,4\%): 1 toxoplasmose chez une PR sous infliximab et 1 uvéite herpétique chez une PR diabétique sous etanercept, MTX et corticoïdes.

- 5 déclarations ne permettaient pas de conclure car les uvéites étaient mal définies.

Les maladies sous-jacentes et les thérapeutiques en cours au moment de l'uvéite sont résumées dans la table III. Sur les 32 déclarations, il n'est jamais précisé si la maladie rhumatismale est active au plan articulaire par ailleurs au moment de l'uvéite.

Dans l'évolution, 9 patients ont vu leur traitement remplacé par une autre biothérapie (table III). Chez 8 autres, le traitement a été stoppé : 5 étanercept, 2 tocilizumab et 1 golimumab. Chez 5 patients, la biothérapie a été suspendue puis reprise après résolution de l'épisode oculaire. Le traitement a été poursuivi à l'identique sans interruption chez 4 patients (1 SPA sous adalimumab et 2 sous étanercept, 1 RP sous infliximab). Dans 6 cas, les données n'étaient pas disponibles. 


\section{Discussion}

Ce travail nous a permis de mettre en évidence la survenue au cours de maladies inflammatoires chroniques traitées par biothérapies et/ou DMARDs d'uvéites de tout type anatomique et d'étiologies variées, incluant les atteintes spécifiques à la maladie et les causes infectieuses.

Sur les 32 uvéites déclarées, seuls 27 cas étaient complètement analysables.

La population de ce registre correspond dans sa présentation globale aux caractéristiques des uvéites rapportées dans la littérature: l'âge moyen est comparable et le sex-ratio est de 0,83, proche du $1 / 1$ retrouvé dans la population uvéitique tout venant ${ }^{7,10-12}$. La proportion des uvéites antérieures de notre population est comparable à la littérature (respectivement $66.7 \%$ versus $66,5 \%)^{14}$. Par contre, 8 des 27 uvéites n'étaient pas des uvéites antérieures : il s'agissait d'uvéites intermédiaires, postérieures ou de panuvéites (1 uvéite intermédiaire et 5 uvéites postérieures). Ces trois types d'uvéite, de fait, ne peuvent pas correspondre à des uvéites rhumatismales qui sont des uvéites antérieures, synéchiantes, uni ou bilatérales et ne sont jamais granulomateuses ${ }^{15-}$ 17. De plus, les panuvéites, jamais attribuables à la maladie rhumatismale, représentaient $7,4 \%$ de notre cohorte; elles représentent $15 \%$ des formes d'uvéites tout venant ${ }^{18}$. A noter, 5 de ces uvéites ont formellement été étiquetées infectieuses: une tuberculose, deux toxoplasmoses oculaires et deux uvéites herpétiques. On peut dès lors se poser la question du rôle propre de la biothérapie et des DMARDS dans l'augmentation du risque infectieux.

Dans notre étude, un seul patient SPA a présenté une authentique tuberculose pulmonaire et oculaire sous forme d'une uvéite postérieure bilatérale avec vascularite au fond d'œil. II s'agissait d'une primo-infection tuberculeuse, sous la forme d'une miliaire avec atteinte ophtalmique, alors que I'IDR était négative à l'introduction de l'étanercept; la rareté de cette complication sous étanercept nous a permis de publier cette observation ${ }^{19}$. Globalement, l'incidence des uvéites tuberculeuses va de 0,39 à $18 \%$ dans la littérature ${ }^{20}$. Elles sont de 2 types : tuberculose maladie par présence du bacille Mycobacterium tuberculosis dans les prélèvements oculaires, et tuberculose latente par réaction immunologique médiée, à distance d'un foyer tuberculeux 
méconnu ou mal traité $e^{20,21}$. Le risque de développer une atteinte oculaire est favorisé par l'immunosuppression liée aux corticostéroïdes et aux autres traitements agissant sur l'immunitée23

De nombreuses études montrent que le risque infectieux, notamment la tuberculose, est augmenté chez les patients atteints de rhumatismes inflammatoires, indépendamment de tout traitement par biothérapies. Les facteurs de risques majeurs identifiés sont : la corticothérapie associée $(R R=2,2$ $[1,5-3,4])$, le tabagisme actif $(R R=1,6[1-2,5])$ et la positivité du facteur rhumatoïde $(R R=2$ [1,3-3]) 20,24,25. Pour la moitié des cas dépistés, la réactivation d'une tuberculose latente sous antiTNFa survient dans la première année avec une proportion de $60 \%$ de tuberculoses extra-pulmonaires, contre $80 \%$ de formes pulmonaires pures dans la population générale ${ }^{25}$.

Deux toxoplasmoses oculaires ont été déclarées sous forme d'une uvéite postérieure bilatérale et d'une panuvéite unilatérale. Dans la population tout venant, la toxoplasmose oculaire est la première cause d'inflammation du segment postérieur en Europe ${ }^{5-7}$. Elle se caractérise par un foyer de rétinochoroïdite au fond d'œil se présentant sous forme d'une lésion blanchâtre, profonde, à bords flous, fréquemment satellite d'une lésion cicatricielle pigmentée et/ou atrophique. Le diagnostic se fait sur l'aspect du fond d'œil et peut être confirmé par PCR de l'humeur aqueuse lors d'une ponction de chambre antérieure ${ }^{27}$.

En cas d'immunodépression, on peut observer des toxoplasmoses oculaires plus sévères ou récidivantes : atteinte multifocale d'un seul œil, inflammation sévère bilatérale ou encore zone de nécrose rétinienne extensive. Ces atteintes peuvent être vues chez le patient $\mathrm{VIH}$ mais également dans les populations traitées par cortisone au long cours et/ou immunosuppresseurs ${ }^{28-30}$.

Deux uvéites herpétiques ont été prouvées dans notre étude. II s'agissait d'une uvéite postérieure et d'une panuvéite. Les infections virales du groupe herpès sont l'étiologie la plus fréquente des uvéites. II s'agit le plus souvent d'une uvéite antérieure, granulomateuse et hypertensive, pouvant s'associer à des manifestations extra-oculaires telles qu'un herpès labial ou une gingivo-stomatite. Le profil sérologique est peu contributif. La ponction de chambre antérieure avec analyse par PCR 
de l'humeur aqueuse peut permettre d'obtenir un diagnostic étiologique ${ }^{31-33}$. Dans notre groupe de patients, la fréquence des uvéites antérieures est très proche de celle de la population générale ${ }^{10-}$ 13. Or, l'origine rhumatismale de l'uvéite est supposée d'emblée sauf dans 5 cas. Dans ce registre déclaratif, la description clinique ophtalmologique n'est pas suffisamment précise dans la majorité des cas pour une interprétation fine des données. Le caractère granulomateux de l'uvéite est indispensable pour le diagnostic car il exclut l'étiologie rhumatismale liée au HLA B27 et oriente soit vers une étiologie infectieuse : virus du groupe herpès (Herpès simplex, CMV, HHV6...) le plus fréquemment, soit vers des granulomatoses telles que la tuberculose, la syphilis ou la sarcoïdose ${ }^{16-18}$. Dans notre étude, aucune donnée anatomique ne précise s'il s'agit d'uvéite synéchiante, qui pourrait correspondre effectivement à une atteinte spécifique, ou s'il s'agit d'une uvéite granulomateuse. Ces données manquantes, inhérentes à ce type de registre déclaratif, constitue une limite à l'interprétation des données. Ceci nous amène à nous demander quelle est la part rhumatismale spécifique et la part infectieuse parmi ces 22 uvéites qui n'ont pas eu de diagnostic étiologique formel. Enfin, l'activité inflammatoire articulaire du rhumatisme sous-jacent n'étant pas précisée, il est difficile de savoir si le patient était en phase d'échappement thérapeutique au moment de l'uvéite.

Les maladies inflammatoires, et notamment la polyarthrite rhumatoïde, sont reconnues comme facteur de risque infectieux en soi, mais les patients sont exposés à un risque supplémentaire lorsqu'ils sont sous agents cytotoxiques qui peuvent induire des neutropénies favorisant indirectement les phénomènes infectieux bactériens, viraux et fungiques ${ }^{34-36}$. Les études tirées du registre CORRONA ont montré une augmentation du risque infectieux sous MTX et sous antiTNFa par rapport aux autres DMARDS ${ }^{36}$. Par contre, il n'y avait pas d'effet cumulatif de l'association MTX et antiTNFa. II s'agissait principalement d'infections à germes communautaires touchant les voies aériennes supérieures ou d'infections virales à VZV ${ }^{31,32,36}$.

Le risque infectieux est plus important sous traitements biologiques : infections à germes banals et candidoses mais également la tuberculose ${ }^{36-44}$. En effet, le risque de réactivation tuberculeuse de 
2 à 4 fois plus important chez la population sous antiTNFa, avec un sur-risque pour les anticorps monoclonaux ${ }^{35}$.

Dans les autres registres de Rhumatologie, le risque viral, notamment l'herpès et le zona, est augmenté quelle que soit la biothérapie et quelle que soit la population étudiée : PR, SPA, AJI ${ }^{44}$. Dans la population générale, les uvéites d'étiologie infectieuse prédominent, herpès principalement. Nous émettons donc l'hypothèse que la plus grande part des uvéites antérieures déclarées sous biothérapie est en fait de mécanisme infectieux, conséquence de l'immunodépression.

Enfin, concernant la thérapeutique, les uvéites n'ont jamais été décrites dans les essais thérapeutiques comme effets secondaires directs des biothérapies ou des DMARDS. Par contre, dans la littérature, il a été évoqué un lien entre le traitement par étanercept et la survenue d'uvéites, lors d'une communication à la Société Française de Rhumatologie (SFR) en 2010, repris en $2014^{45}$. Cet article suggérait une majoration du risque d'uvéite dans la première année de traitement par étanercept versus adalimumab, mais ce risque demeurait identique pour les autres biothérapies $^{45}$. Un lien entre étanercept et sclérite a également été évoqué ${ }^{46}$. Ces hypothèses n'ont jamais été confirmées et d'autres études ont montré depuis que l'incidence des uvéites n'était pas plus importante sous étanercept que sous une autre biothérapie ${ }^{47-49}$.

Enfin, les biothérapies et en particulier les anti TNFa sont actuellement utilisés dans le traitement des uvéites postérieures non infectieuses, notamment la maladie de Behçet, avec une RBU (Référentiels de Bon Usage hors GHS) pour infliximab, étanercept et adalimumab ${ }^{50-53}$.

\section{Conclusion}

La présence d'une uvéite sous biothérapie ne doit pas faire seulement évoquer un échappement thérapeutique, surtout si la manifestation oculaire est isolée. L'hypothèse infectieuse favorisée par la biothérapie doit être discutée systématiquement. L'identification de l'étiologie d'une uvéite survenant sous biothérapie est cruciale pour la prise en charge du patient. 


\section{ANNEXES}

\section{Table I - Principaux critères de classification des uvéites}

\begin{tabular}{|c|c|c|}
\hline Classification selon le terrain & Âge du patient & Uvéites de l'enfant, de l'adulte ou du sujet âgé \\
\hline Classification selon l'étiologie & \multicolumn{2}{|c|}{$\begin{array}{l}\text { Infection et/ou inflammation } \\
\text { Maladie systémique ou uvéite isolée }\end{array}$} \\
\hline \multirow{9}{*}{$\begin{array}{l}\text { Classification selon l'examen } \\
\text { ophtalmologique }\end{array}$} & Localisation & Antérieure, intermédiaire, postérieure, panuvéite \\
\hline & & Uni- ou bilatérale (concomitante ou " à bascule ") \\
\hline & Mode évolutif & Aigu ou chronique \\
\hline & Intensité & Chambre antérieure : tyndall cellulaire et flare \\
\hline & & Vitré : haze et cellularité vitréenne \\
\hline & Caractère " granulomateux " & Présentation des précipités rétrodescemétiques \\
\hline & & Autres éléments « granulomateux » \\
\hline & Complications anatomiques & Synéchies, hypertonie, œdème papillaire \pm maculaire \\
\hline & Complications fonctionnelles & Retentissement de l'uvéite sur la vision \\
\hline
\end{tabular}

Figure 1 - Répartition des uvéites déclarées à la pharmacovigilance
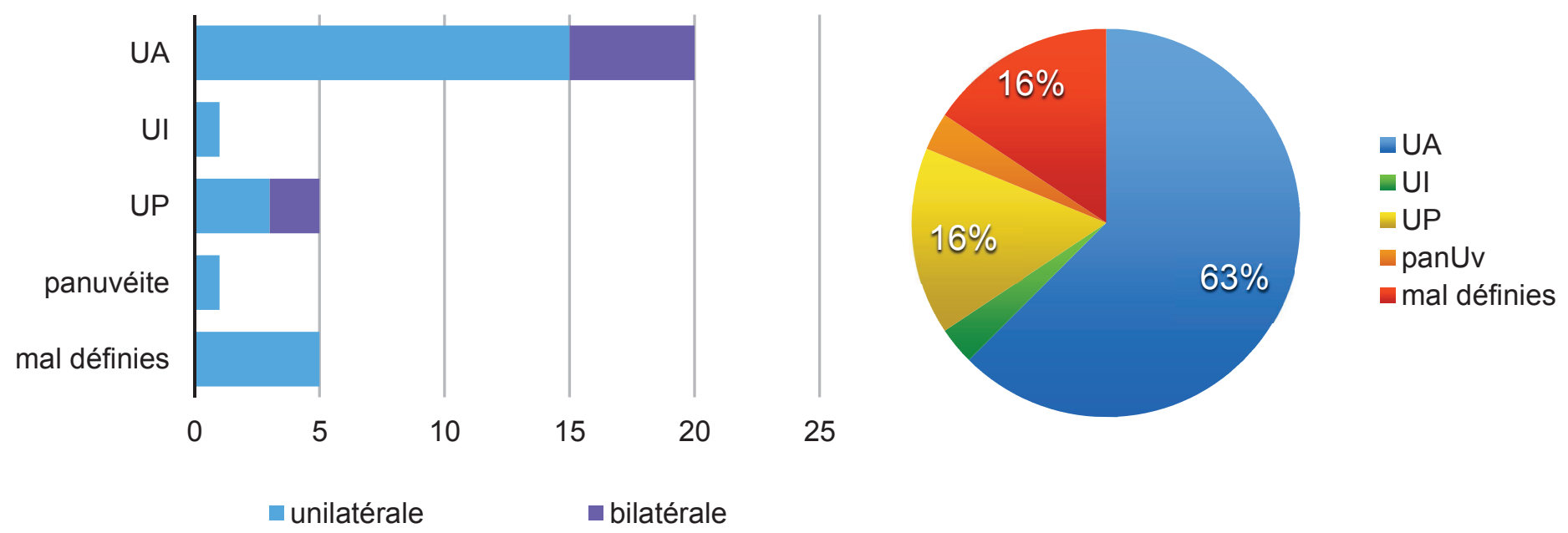

UA : uvéite antérieure

UI : uvéite intermédiaire

UP : uvéite postérieure

PanUV : panuvéite 
Table II - Caractéristiques des patients

\begin{tabular}{|c|c|c|c|c|c|}
\hline & Sexe & Age & Antécédents & maladie sous-jacente & traitement \\
\hline 1 & $M$ & $43 \mathrm{~A}$ & $\begin{array}{l}\text { Dyslipidémie } \\
\text { Hypertension artérielle }\end{array}$ & $\begin{array}{l}\text { Spondylarthrite } \\
\text { ankylosante }\end{array}$ & étanercept 50 \\
\hline 2 & $F$ & $46 \mathrm{~A}$ & $\begin{array}{l}\text { Cancer du poumon } \\
\text { métastatique }\end{array}$ & Syndrome lupique & Plaquenil 200 2x/j \\
\hline 3 & $M$ & $30 \mathrm{~A}$ & Spondylarthrite ankylosante & $\begin{array}{l}\text { Spondylarthrite } \\
\text { ankylosante }\end{array}$ & tocilizumab \\
\hline 4 & $F$ & $49 \mathrm{~A}$ & Polyarthrite rhumatoïde & $\begin{array}{l}\text { Polyarthrite } \\
\text { rhumatoïde }\end{array}$ & $\begin{array}{l}\text { étanercept } 50 \text {, leflunomide } \\
20 \mathrm{mg} / \mathrm{j}\end{array}$ \\
\hline 5 & M & $60 \mathrm{~A}$ & HTA, dyslipidémie, IDM stenté & $\begin{array}{l}\text { Spondylarthrite } \\
\text { ankylosante }\end{array}$ & adalimumab 40 \\
\hline 6 & $F$ & $38 \mathrm{~A}$ & Cholécystectomie, Colectomie & $\begin{array}{l}\text { rhumatisme } \\
\text { inflammatoire inclassé }\end{array}$ & infliximab \\
\hline 7 & $M$ & $51 \mathrm{~A}$ & & $\begin{array}{l}\text { rhum inflammatoire } \\
\text { inclassé }\end{array}$ & étanercept 50 \\
\hline 8 & $F$ & $52 \mathrm{~A}$ & $\begin{array}{l}\text { Spondylarthrite } \\
\text { ankylosante }\end{array}$ & $\begin{array}{l}\text { Spondylarthrite } \\
\text { ankylosante }\end{array}$ & étanercept 50 \\
\hline 9 & M & $60 \mathrm{~A}$ & $\begin{array}{l}\text { Hypertension artérielle } \\
\text { Hyperuricémie, insuffisance } \\
\text { rénale }\end{array}$ & $\begin{array}{l}\text { Spondylarthrite } \\
\text { ankylosante }\end{array}$ & golimumab 50 \\
\hline 10 & $M$ & $45 \mathrm{~A}$ & & Psoriasis & étanercept 50 \\
\hline 11 & $F$ & $59 \mathrm{~A}$ & Spondylarthrite ankylosante & $\begin{array}{l}\text { Spondylarthrite } \\
\text { ankylosante }\end{array}$ & infliximab \\
\hline 12 & $\mathrm{~F}$ & $31 \mathrm{~A}$ & Hypertension artérielle & Maladie de Crohn & adalimumab 40 \\
\hline 13 & $M$ & $30 \mathrm{~A}$ & Hypertension artérielle & $\begin{array}{l}\text { rhumatisme } \\
\text { psoriasique }\end{array}$ & infliximab \\
\hline 14 & $F$ & $61 \mathrm{~A}$ & Diabète sucré de type II & $\begin{array}{l}\text { Polyarthrite } \\
\text { rhumatoïde }\end{array}$ & étanercept 50, MTX, CTO \\
\hline 15 & $F$ & $37 \mathrm{~A}$ & Spondylarthrite ankylosante & $\begin{array}{l}\text { Spondylarthrite } \\
\text { ankylosante }\end{array}$ & étanercept 50 \\
\hline 16 & $M$ & $20 \mathrm{~A}$ & $\mathrm{MICl}$ & $\mathrm{RCH}$ Corticorésistante & infliximab \\
\hline 17 & $F$ & $53 \mathrm{~A}$ & Polyarthrite rhumatoïde & Polyarthrite rhumatoïde & étanercept 50 \\
\hline 18 & $F$ & NA & Polyarthrite rhumatoïde & $\begin{array}{l}\text { Polyarthrite } \\
\text { rhumatoïde }\end{array}$ & étanercept 50 \\
\hline
\end{tabular}


Table II - Caractéristiques des patients

\begin{tabular}{|c|c|c|c|c|c|}
\hline & Sexe & Age & Antécédents & maladie sous-jacente & traitement \\
\hline 19 & $F$ & $31 \mathrm{~A}$ & $\mathrm{MICl}$ & Maladie de Crohn & infliximab 340 \\
\hline 20 & M & $36 \mathrm{~A}$ & Spondylarthrite ankylosante & $\begin{array}{l}\text { Spondylarthrite } \\
\text { ankylosante }\end{array}$ & étanercept $252 x / s$ \\
\hline 21 & $F$ & $46 \mathrm{~A}$ & Polyarthrite rhumatoïde & $\begin{array}{l}\text { Polyarthrite } \\
\text { rhumatoïde neg }\end{array}$ & étanercept 25 \\
\hline 22 & M & $19 \mathrm{~A}$ & Spondylarthrite ankylosante & $\begin{array}{l}\text { Spondylarthrite } \\
\text { ankylosante }\end{array}$ & $\begin{array}{l}\text { étanercept } 25 \text {, } \\
\text { sulfasalazine } 5003 x / j\end{array}$ \\
\hline 23 & $F$ & $47 \mathrm{~A}$ & $\begin{array}{l}\text { rhumatisme inflammatoire } \\
\text { inclassé }\end{array}$ & $\begin{array}{l}\text { rhumatisme } \\
\text { inflammatoire inclassé }\end{array}$ & $\begin{array}{l}\text { tocilizumab, MTX 20, СТO } \\
5 \mathrm{mg} / \mathrm{j}\end{array}$ \\
\hline 24 & $F$ & $67 \mathrm{~A}$ & Allergie médicamenteuse & $\begin{array}{l}\text { Polyarthrite } \\
\text { rhumatoïde }\end{array}$ & MTX 20, CTO 10mg/j \\
\hline 25 & M & $39 \mathrm{~A}$ & Spondylarthrite ankylosante & $\begin{array}{l}\text { Spondylarthrite } \\
\text { ankylosante }\end{array}$ & étanercept 50 \\
\hline 26 & M & $39 \mathrm{~A}$ & Spondylarthrite ankylosante & $\begin{array}{l}\text { Spondylarthrite } \\
\text { ankylosante }\end{array}$ & étanercept 50 \\
\hline 27 & M & $28 \mathrm{~A}$ & Spondylarthrite ankylosante & $\begin{array}{l}\text { Spondylarthrite } \\
\text { ankylosante }\end{array}$ & étanercept 50 \\
\hline 28 & $F$ & $32 \mathrm{~A}$ & Polyarthrite rhumatoïde & $\begin{array}{l}\text { Polyarthrite } \\
\text { rhumatoïde }\end{array}$ & $\begin{array}{l}\text { étanercept } 50 \text {, leflunomide } \\
20 \text {, CTO } 4 \mathrm{mg} / \mathrm{j}\end{array}$ \\
\hline 29 & $F$ & $65 \mathrm{~A}$ & Polyarthrite rhumatoïde & $\begin{array}{l}\text { Polyarthrite } \\
\text { rhumatoïde }\end{array}$ & infliximab \\
\hline 30 & $F$ & $54 \mathrm{~A}$ & Polyarthrite rhumatoïde & Polyarthrite rhumatoïde & $\begin{array}{l}\text { tocilizumab, MTX } \\
20 \mathrm{mg} / \mathrm{sem}\end{array}$ \\
\hline 31 & M & $24 \mathrm{~A}$ & Spondylarthrite ankylosante & $\begin{array}{l}\text { Spondylarthrite } \\
\text { ankylosante }\end{array}$ & étanercept 50 \\
\hline 32 & $M$ & $70 A$ & diabète, Insuffisance rénale & goutte polyarticulaire & anakinra \\
\hline
\end{tabular}

$\mathrm{CO}=$ corticothérapie orale, $\mathrm{MTX}=$ méthotrexate 
Table III - Description clinique des uvéites

\begin{tabular}{|c|c|c|c|}
\hline & type d'uvéite & maladie sous jacente & $\begin{array}{l}\text { traitement en cours et } \\
\text { évolution du traitement }\end{array}$ \\
\hline 1 & UA OG, hypertensive & Spondylarthrite ankylosante & adalimumab $\rightarrow$ poursuite \\
\hline 2 & UA OD, oedème maculaire & Spondylarthrite ankylosante & golimumab $\rightarrow$ stop \\
\hline 3 & UA OG & Spondylarthrite ankylosante & étanercept $\rightarrow$ adalimumab \\
\hline 4 & UA OG & Spondylarthrite ankylosante & étanercept $\rightarrow$ adalimumab \\
\hline 5 & UA OG & Spondylarthrite ankylosante & étanercept $\rightarrow$ poursuite \\
\hline 6 & UA OG & Spondylarthrite ankylosante & étanercept $\rightarrow$ poursuite \\
\hline 7 & $\begin{array}{l}\text { UA OD, synéchies, non } \\
\text { granulomateuse }\end{array}$ & Spondylarthrite ankylosante & étanercept $\rightarrow$ adalimumab \\
\hline 8 & $\begin{array}{l}\text { UA OD, hypopion, synéchies, } \\
\text { non granulomateuse }\end{array}$ & Spondylarthrite ankylosante & étanercept + sulfasalazine \\
\hline 9 & UA OG, oedème papillaire & PR sérodéfinie & $\begin{array}{l}\text { tocilizumab + MTX 20mg } \\
\rightarrow \text { stop }\end{array}$ \\
\hline 10 & UA OG à œil blanc, chronique & PR non sérodéfinie & étanercept $\rightarrow$ stop \\
\hline 11 & UA OD & $\begin{array}{l}\text { Rhumatisme inflammatoire } \\
\text { inclassé }\end{array}$ & infliximab $\rightarrow$ suspendu \\
\hline 12 & UA bilatérale, synéchies & Spondylarthrite ankylosante & étanercept $\rightarrow$ infliximab \\
\hline 13 & $\begin{array}{l}\text { UA bilatérale, granulomateuse, } \\
\text { hypertensive, nodules de } \\
\text { Koeppe }\end{array}$ & PR sérodéfinie & étanercept $\rightarrow$ stop \\
\hline 14 & $\begin{array}{l}\text { UA bilatérale non } \\
\text { granulomateuse }\end{array}$ & PR sérodéfinie & $\begin{array}{l}\text { étanercept + leflunomide } \\
\rightarrow \text { certolizumab }\end{array}$ \\
\hline 15 & $\begin{array}{l}\text { UA bilatérale, non } \\
\text { granulomateuse, synéchies }\end{array}$ & PR sérodéfinie & $\begin{array}{l}\text { étanercept + leflunomide + } \\
\text { CTO } \rightarrow \text { stop }\end{array}$ \\
\hline 16 & UA bilatérale, synéchies & $\begin{array}{l}\text { Rhumatisme inflammatoire } \\
\text { inclassé }\end{array}$ & étanercept $\rightarrow$ leflunomide \\
\hline 17 & $\begin{array}{l}\text { UA bilatérale, oedème } \\
\text { maculaire }\end{array}$ & Psoriasis & étanercept $\rightarrow$ suspendu \\
\hline 18 & $\begin{array}{l}\text { UA bilatérale, hypertensive, } \\
\text { hypopion }\end{array}$ & Maladie de Crohn & adalimumab \\
\hline 19 & UA bilatérale & Rectocolite hémorragique & infliximab \\
\hline
\end{tabular}




\begin{tabular}{|c|c|c|c|}
\hline & type d'uvéite & maladie sous jacente & $\begin{array}{l}\text { traitement en cours et } \\
\text { évolution du traitement }\end{array}$ \\
\hline 20 & UI OD, hyalite & $\begin{array}{l}\text { Rhumatisme inflammatoire } \\
\text { inclassé }\end{array}$ & $\begin{array}{l}\text { tocilizumab + MTX } 20 \mathrm{mg}+ \\
\text { CTO } \rightarrow \text { suspendu }\end{array}$ \\
\hline 21 & UP unilatérale & Spondylarthrite ankylosante & tocilizumab $\rightarrow$ stop \\
\hline 22 & $\begin{array}{l}\text { UP unilatérale, oedème } \\
\text { maculaire }\end{array}$ & Spondylarthrite ankylosante & infliximab $\rightarrow$ golimumab \\
\hline 23 & $\begin{array}{l}\text { UP OG, synéchies, sclérite } \\
\text { postérieure }\end{array}$ & PR sérodéfinie & étanercept $\rightarrow$ infliximab \\
\hline 24 & UP bilatérale, vascularite au FO & Spondylarthrite ankylosante & étanercept $\rightarrow$ stop \\
\hline 25 & $\begin{array}{l}\text { UP bilatérale, foyers de } \\
\text { choriorétinite, nécrose }\end{array}$ & Goutte polyarticulaire & anakinra $\rightarrow$ suspendu \\
\hline 26 & $\begin{array}{l}\text { panuvéite unilatérale } \\
\text { granulomateuse, hypertensive }\end{array}$ & PR sérodéfinie & $\begin{array}{l}\text { étanercept + MTX + CTO } \\
\rightarrow \text { stop }\end{array}$ \\
\hline 27 & $\begin{array}{l}\text { panuvéite OD, PCR } \\
\text { toxoplasmose positive dans le } \\
\text { vitrée }\end{array}$ & PR sérodéfinie & infliximab $\rightarrow$ suspendu \\
\hline
\end{tabular}

MTX : méthotrexate, CTO : corticothérapie orale 


\section{Références}

1. Durrani OM, Tehrani NN, Marr JE, Moradi P, Stavrou P, Murray PI. Degree, duration, and causes of visual loss in uveitis. Br J Ophthalmol. 2004 Sep;88(9):1159-62.

2. Durrani OM, Meads CA, Murray PI. Uveitis: a potentially blinding disease. Ophthalmologica. 2004 Jul-Aug;218(4):223-36. Review.

3. Rosenbaum JT. Uveitis in spondyloarthritis including psoriatic arthritis, ankylosing spondylitis, and inflammatory bowel disease. Clin Rheumatol. 2015 Jun;34(6):999-1002. doi: 10.1007/s10067-015-2960-8.

4. Stolwijk C, van Tubergen A, Castillo-Ortiz JD, Boonen A. Prevalence of extra-articular manifestations in patients with ankylosing spondylitis: a systematic review and meta-analysis. Ann Rheum Dis. 2015 Jan;74(1):65-73.

5. Bodaghi B, Wechsler B, Du-Boutin LT, Cassoux N, LeHoang P, Piette JC. [Chronic severe uveitis: classification, search for etiology and therapeutic approach].Rev Med Interne. 2003 Dec;24(12):794-802.

6. Grajewski RS, Caramoy A, Frank KF, Rubbert-Roth A, Fätkenheuer G, Kirchhof B, Cursiefen C, Heindl LM. Spectrum of Uveitis in A German Tertiary Center: Review of 474 Consecutive Patients. Ocul Immunol Inflamm. 2015 Mar 11:1-7.

7. Jakob E, Reuland MS, Mackensen F, Harsch N, Fleckenstein M, Lorenz HM, Max R, Becker MD. Uveitis subtypes in a german interdisciplinary uveitis center--analysis of 1916 patients. J Rheumatol. 2009 Jan;36(1):127-36.

8. Nguyen AM, Sève P, Le Scanff J, Gambrelle J, Fleury J, Broussolle C, Grange JD, Kodjikian L. [Clinical and etiological aspects of uveitis: a retrospective study of 121 patients referred to a tertiary centre of ophthalmology]. Rev Med Interne. 2011 Jan;32(1):9-16.

9. Bloch-Michel E, Nussenblatt RB. International Uveitis Study Group recommendations for the evaluation of intraocular inflammatory disease. Am J Ophthalmol. 1987 Feb15;103(2):234-5.

10. Reeves SW, Sloan FA, Lee PP, Jaffe GJ. Uveitis in the elderly: epidemiological data from the National Long-term Care Survey Medicare Cohort. Ophthalmology. 2006 Feb;113(2):307.

11. Bajwa A, Osmanzada D, Osmanzada S, Khan I, Patrie J, Xin W, Reddy AK. Epidemiology of uveitis in the mid-Atlantic United States. Clinical Ophthalmology (Auckland, N.Z.). 1/01/01 00:00; 9: 889-901

12. Acharya NR, Tham VM, Esterberg E, Borkar DS, Parker JV, Vinoya AC, Uchida A. Incidence and prevalence of uveitis: results from the Pacific Ocular Inflammation Study. JAMA Ophthalmol. 2013 Nov;131(11):1405-12.

13. Gritz DC, Wong IG. Incidence and prevalence of uveitis in Northern California;the Northern California Epidemiology of Uveitis Study. Ophthalmology. 2004 Mar;111(3):491-500;

14. McCannel CA, Holland GN, Helm CJ, Cornell PJ, Winston JV, Rimmer TG. Causes of uveitis in the general practice of ophthalmology. UCLA Community-Based Uveitis Study Group. Am J Ophthalmol. 1996 Jan;121(1):35-46.

15. Zarco P, González CM, Rodríguez de la Serna A, Peiró E, Mateo I, Linares L, Calvo J, CeaCalvo L, Arteaga MJ, Vanaclocha F, Marín-Jiménez I, García-Vicuña R. Extra-articular disease in patients with spondyloarthritis. Baseline characteristics of the spondyloarthritis cohort of the AQUILES study. Reumatol Clin. 2015 Mar-Apr;11(2):83-9.

16. Chang JH, McCluskey PJ, Wakefield D. Acute anterior uveitis and HLA-B27. Surv Ophthalmol. 2005 Jul-Aug;50(4):364-88. Review.

17. Monnet D, Breban M, Hudry C, Dougados M, Brézin AP. Ophthalmic findings and frequency of extraocular manifestations in patients with HLA-B27 uveitis: a study of 175 cases. Ophthalmology. 2004 Apr;111(4):802-9.

18. Etude ULISSE : difficultés étiologiques dans les uvéites

19. Cabane L, Baillif S, Asrarguis G, Gastaud P, Queyrel V, Tieulié N. Uveitis associated with spondylitis: It may be tuberculosis!. J Fr Ophtalmol. 2016 Apr;39(4):e83-4

20. Gupta A, Sharma A, Bansal R, Sharma K. Classification of intraocular tuberculosis. Ocul Immunol Inflamm. 2015 Feb;23(1):7-13.

21. Gupta V, Shoughy SS, Mahajan S, Khairallah M, Rosenbaum JT, Curi A, Tabbara KF. Clinics of ocular tuberculosis. Ocul Immunol Inflamm. 2015 Feb;23(1):14-24. 
22. Ponce de Leon D, Acevedo-Vasquez E, Alvizuri S, Gutierrez C, Cucho M, Alfaro J, Perich R, Sanchez-Torres A, Pastor C, Sanchez-Schwartz C, Medina M, Gamboa R, Ugarte M. Comparison of an interferon-gamma assay with tuberculin skin testing for detection of tuberculosis (TB) infection in patients with rheumatoid arthritis in a TB-endemic population. $J$ Rheumatol. 2008 May;35(5):776-81.

23. Ang M, Htoon HM, Chee SP. Diagnosis of tuberculous uveitis: clinical application of an interferon-gamma release assay. Ophthalmology. 2009 Jul;116(7):1391-6.

24. Van der Veen MJ, van der Heide A, Kruize AA, Bijlsma JW. Infection rate and use of antibiotics in patients with rheumatoid arthritis treated with methotrexate. Ann Rheum Dis. 1994 Apr;53(4):224-8.

25. Djebbar M. infection risk when receiving anti TNF treatment: crucial and major concern or not so bad reality ? Rev Mar Rhum 2015; 31 : 34-44

26. Rodrigues KF, Faria e Arantes TE, Muccioli C, Neto JL, Pinheiro MM. Incidence of Toxoplasma retinochoroiditis in patients with ankylosing spondylitis after using TNF- $\alpha$ blockers. Parasitol Int. 2013 Jun;62(3):272-5.

27. Lin SJ, Lee SE, Kim SH, Hong SH, You YS, Kwon OW, Kim HS. Prevalence of Toxoplasma gondii and Toxocara canis among patients with uveitis. Ocul Immunol Inflamm. 2014 Oct;22(5):360-6.

28. Lassoued S, Zabraniecki L, Marin F, Billey T. Toxoplasmic chorioretinitis and antitumor necrosis factor treatment in rheumatoid arthritis. Semin Arthritis Rheum. 2007 Feb;36(4):262-3.

29. Smith JR, Cunningham ET Jr. Atypical presentations of ocular toxoplasmosis. Curr Opin Ophthalmol. 2002 Dec;13(6):387-92. Review.

30. Nicholson $\mathrm{DH}$, Wolchok EB. Ocular toxoplasmosis in an adult receiving long-term corticosteroid therapy. Arch Ophthalmol. 1976 Feb;94(2):248-54.

31. Strangfeld A, Listing J, Herzer P, Liebhaber A, Rockwitz K, Richter C, Zink A. Risk of herpes zoster in patients with rheumatoid arthritis treated with anti-TNF-alpha agents. JAMA. 2009 Feb 18;301(7):737-44.

32. Serac G, Tubach F, Mariette $X$, Salmon-Céron D, Ravaud P, Lioté F, Laharie D, Ziza JM, Marguerie L, Bonnet C, Falgarone G, Nicolas N, Lortholary O, Chosidow O. Risk of herpes zoster in patients receiving anti-TNF- $\alpha$ in the prospective French RATIO registry. $\mathrm{J}$ Invest Dermatol. 2012 Mar;132(3 Pt 1):726-9.

33. Che H, Lukas C, Morel J, Combe B. Risk of herpes/herpes zoster during anti-tumor necrosis factor therapy in patients with rheumatoid arthritis. Systematic review and meta-analysis. Joint Bone Spine. 2014 May;81(3):215-21.

34. Franklin J, Lunt M, Bunn D, Symmons D, Silman A. Risk and predictors of infection leading to hospitalisation in a large primary-care-derived cohort of patients with inflammatory polyarthritis. Ann Rheum Dis. 2007 Mar;66(3):308-12.

35. Emery P, Gallo G, Boyd H, Morgan CL, Currie CJ, Poole CD, Nab HW. Association between disease activity and risk of serious infections in subjects with rheumatoid arthritis treated with etanercept or disease-modifying anti-rheumatic drugs. Clin Exp Rheumatol. 2014 SepOct;32(5):653-60. Epub 2014 Sep 5.

36. Greenberg JD, Reed G, Kremer JM, Tindall E, Kavanaugh A, Zheng C, Bishai W, Hochberg $\mathrm{MC}$; CORRONA Investigators. Association of methotrexate and tumour necrosis factor antagonists with risk of infectious outcomes including opportunistic infections in the CORRONA registry. Ann Rheum Dis. 2010 Feb;69(2):380-6.

37. Haerter G, Manfras BJ, de Jong-Hesse Y, Wilts H, Mertens T, Kern P, Schmitt M. Cytomegalovirus retinitis in a patient treated with anti-tumor necrosis factor alpha antibody therapy for rheumatoid arthritis. Clin Infect Dis. 2004 Nov 1;39(9):e88-94.

38. Segal BH, Sneller MC. Infectious complications of immunosuppressive therapy in patients with rheumatic diseases. Rheum Dis Clin North Am. 1997 May;23(2):219-37. Review.

39. Mikuls TR, Moreland LW. Benefit-risk assessment of infliximab in the treatment of rheumatoid arthritis. Drug Saf. 2003;26(1):23-32. Review.

40. Wiland P, Głowska A, Chlebicki A, Szechiński J. [Analysis of efficacy and safety of multiple intravenous infusion of anti-tumor necrosis factor-alpha monoclonal antibody (Remicade) combined with methotrexate compared with sodium aurothiomalate and intramuscular depot 
methylprednisolone in rheumatoid arthritis]. Pol Arch Med Wewn. 2002 Nov;108(5):1055-63. Polish.

41. Bongartz T, Sutton AJ, Sweeting MJ, Buchan I, Matteson EL, Montori V. Anti-TNF antibody therapy in rheumatoid arthritis and the risk of serious infections and malignancies: systematic review and meta-analysis of rare harmful effects in randomized controlled trials. JAMA. 2006 Jun 7;295(21):2482.

42. Engelhard SB, Haddad Z, Bajwa A, Patrie J, Xin W, Reddy AK. Infectious uveitis in Virginia. Clin Ophthalmol. 2015;9:1589-94.

43. Lim WS, Powell RJ, Johnston ID. Tuberculosis and treatment with infliximab. N Engl J Med. 2002 Feb 21;346(8):623-6.

44. Ruderman EM. Overview of safety of non-biologic and biologic DMARDs. Rheumatology (Oxford). 2012 Dec;51 Suppl 6:vi37-43.

45. Wendling $D^{1}$, Joshi $A$, Reilly $P$, Jalundhwala $Y J$, Mittal $M$, Bao Y. Comparing the risk of developing uveitis in patients initiating anti-tumor necrosis factor therapy for ankylosing spondylitis: an analysis of a large US claims database. Curr Med Res Opin. 2014 Dec;30(12):2515-21.

46. Gaujoux-Viala C, Giampietro C, Gaujoux T, Ea HK, Prati C, Orcel P, Wendling D, Lioté F. Scleritis: a paradoxical effect of etanercept? Etanercept-associated inflammatory eye disease. J Rheumatol. 2012 Apr;39(4):881.

47. Lim LL, Fraunfelder FW, Rosenbaum JT. Do tumor necrosis factor inhibitors cause uveitis? A registry-based study. Arthritis Rheum. 2007 Oct;56(10):3248-52.

48. Otten MH, Anink J, Prince FH, Twilt M, Vastert SJ, ten Cate R, Hoppenreijs EP, Armbrust W, Gorter SL, van Pelt PA, Kamphuis SS, Dolman KM, Swart JF, van den Berg JM, KoopmanKeemink Y, van Rossum MA, Wulffraat NM, van Suijlekom-Smit LW. Trends in prescription of biological agents and outcomes of juvenile idiopathic arthritis: results of the Dutch national Arthritis and Biologics in Children Register. Ann Rheum Dis. 2015 Jul;74(7):1379-86.

49. Kearsley-Fleet L, Davies R, Lunt M, Southwood TR, Hyrich KL. Factors associated with improvement in disease activity following initiation of etanercept in children and young people with Juvenile Idiopathic Arthritis: results from the British Society for Paediatric and Adolescent Rheumatology Etanercept Cohort Study. Rheumatology (Oxford). 2016 May;55(5):840-7

50. Feurer E, Bielefeld P, Saadoun D, Sève P. [Biologics in uveitis]. Rev Med Interne. 2015 Feb;36(2):107-16.

51. Abad S, Sève P, Dhote R, Brézin AP. [Guidelines for the management of uveitis in internal medicine]. Rev Med Interne. 2009 Jun;30(6):492-500.

52. Desbois AC, Addimanda O, Bertrand A, Deroux A, Pérard L, Depaz R, Hachulla E, Lambert M, Launay D, Subran B, Ackerman F, Mariette X, Cohen F, Marie I, Salvarini C, Cacoub P, Saadoun D. Efficacy of Anti-TNFa in Severe and Refractory Neuro-Behcet Disease: An Observational Study. Medicine (Baltimore). 2016 Jun;95(23):e3550

53. AFFSAPS - Référentiels de Bon Usage hors GHS AntiTNF. MAJ Novembre 2012 


\section{Le Serment d'Hippocrate}

Je promets et je jure d'être fidèle aux lois de l'honneur et de la probité dans l'exercice de la Médecine.

Je respecterai toutes les personnes, leur autonomie et leur volonté, sans discrimination.

J'interviendrai pour les protéger si elles sont vulnérables ou menacées dans leur intégrité ou leur dignité. Même sous la contrainte, je ne ferai pas usage de mes connaissances contre les lois de l'humanité.

J'informerai les patients des décisions envisagées, de leurs raisons et de leurs conséquences. Je ne tromperai jamais leur confiance.

Je donnerai mes soins à l'indigent et je n'exigerai pas un salaire au dessus de mon travail.

Admis dans l'intimité des personnes, je tairai les secrets qui me seront confiés et ma conduite ne servira pas à corrompre les mœurs.

Je ferai tout pour soulager les souffrances. Je ne prolongerai pas abusivement la vie ni ne provoquerai délibérément la mort.

Je préserverai l'indépendance nécessaire et je n'entreprendrai rien qui dépasse mes compétences. Je perfectionnerai mes connaissances pour assurer au mieux ma mission.

Que les hommes m'accordent leur estime si je suis fidèle à mes promesses. Que je sois couvert d'opprobre et méprisé si j'y manque. 